Giuseppe Cavaliere

Limited time series with a unit root

Serie Ricerche 2003, n.1

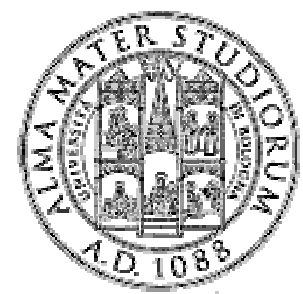

Dipartimento di Scienze Statistiche "Paolo Fortunati" Università degli studi di Bologna 


\title{
Limited time series with a unit root
}

\author{
Giuseppe Cavaliere \\ Dipartimento di Scienze Statistiche, Università di Bologna.
}

\begin{abstract}
This paper develops an asymptotic theory for integrated and near-integrated time series whose range is constrained in some ways. Such a framework arises when integration and cointegration analysis are applied to persistent series which are bounded either by construction or because they are subject to control. The asymptotic properties of some commonly used integration tests are discussed; the bounded unit root distribution is introduced to describe the limiting distribution of the first-order autoregressive coefficient of a random walk under range constraints. The theoretical results show that the presence of such constraints can lead to drastically different asymptotics. Since deviations from the standard unit root theory are measured through noncentrality parameters, simple measures of the impact of range constraints on the asymptotic distributions are obtained. Finally, the proposed asymptotic framework provides an extremely adequate approximation of the finite sample properties of the unit root statistics under range constraints.
\end{abstract}




\section{Introduction}

Despite the extensive literature on modeling non-stationary economic time series (see e.g. Engle and Granger, 1991, Banerjee et al., 1993, and references therein) and limited-dependent variables (see e.g. Maddala, 1986, Hsiao et al., 1999), a controversial and rarely discussed topic is how to interpret and analyze time series whose behavior can be well approximated by means of integrated processes (I(1)) but are 'limited' in the sense that their range is constrained in some ways. Common cases arise in the context of composition ratios, such as expenditure shares or unemployment rates, or in the presence of nonnegativity restrictions, e.g. for nominal interest rates. Moreover, range constraints represent the standard framework in the context of target zone exchange rates and, more generally, of time series which are subject to control. In the following, time series satisfying (onesided or two-sided) range restrictions are called 'limited' or 'bounded'.

Although limited time series cannot be integrated in the usual sense $^{1}$, in various theoretical and econometric contexts they are often modelled in the I(1) framework. For example, several empirical models of European Monetary System exchange rates have been specified in the framework of the (co-)integrated VAR model without taking account of the presence of target zones (see, among many others, Anthony and MacDonald, 1998; Svensson, 1993; see also Phillips, 2001). Similarly, in their influential paper Nelson and Plosser (1982) reject the unit root hypothesis in the U.S. unemployment rate (see the discussions in Caner and Hansen, 2001, and Abadir and Taylor, 1999), while several authors have looked for possible cointegrating relations

\footnotetext{
${ }^{1} \mathrm{~A}$ partial attempt to define $\mathrm{I}(1)$ processes with range constraints is made by Barr and Cuthbertson (1991, note 32), regarding investment shares. They observe that " $(. .$.$) although shares are \mathrm{I}(1)$ in the data there is a theoretical problem in that shares are bounded. Shares cannot be a random walk since such a series is unbounded. However, a random walk is a very special case of an I(1) series, namely linear with an additive Gaussian error. Near the boundary shares must have a non-Gaussian error. Similar considerations apply to variables such as the percentage unemployment and bilateral exchange rates (which are bounded below) which have been examined using cointegration techniques".
} 
linking unemployment rates to other variables. Moreover, some theoretical models consistent with a unit root in the unemployment rate have been proposed (see e.g. Blanchard and Summers, 1986; Lindbeck and Snower, 1989). What is not generally discussed in the literature is (i) why in some cases the constraints can reasonably be neglected and therefore standard I(1) modelling is still appropriate, (ii) to what extent the misspecification error depending on the omission of the range constraints affects standard (co-)integration tests and (iii) how to test for unit roots (or co-integration) in the presence of range constraints.

The existence of range constraints makes the interpretation of the outcome of unit root tests controversial. Suppose e.g. that the I(1) hypothesis is rejected. Should such a rejection be attributed to the presence of $\mathrm{I}(d)$ dynamics, $|d|<1$, or does it depend on the existing constraints? This question is extremely important, especially since in the literature the $I(1)$ hypothesis is usually rejected when applied to limited time series, e.g. unemployment rates or nominal interest rates, while it is not rejected in the great majority of empirical applications to unlimited time series.

In this paper all these issues will be addressed within a unified framework. This aim will be achieved by developing a new asymptotic theory which accounts for both nonstationarity and range restrictions. This approach allows to generalize the large-sample theory for integrated and near-integrated processes to the case of range constraints. The standard unit root distribution can be extended to the case of range constraints; standard theory, see e.g. Phillips (1987a, 1987b), is obtained as a special case. The limit 'bounded unit root' distribution depends on two non-centrality parameters which are expressed in terms of the position of the range limits. Such parameters allow a rapid evaluation of the impact of range constraints on the properties of unit root tests. Moreover, they allow to extend unit root inference to the context of limited time series.

The paper is organized as follows. The next section defines integrated and near-integrated processes under range constraints and some basic asymptotic results are derived. In Section 3 the bounded 
unit root distribution is derived and analyzed in the context of unit root statistics. Implications for unit root tests are discussed in Section 4. In Section 5 the problem of testing for unit roots in the presence of range constraints is tackled. Two illustrative applications are reported in Section 6. Section 7 concludes with a discussion on some extensions of the results obtained.

\section{Limited autoregressive processes}

We start by considering a standard, integrated or near-integrated (NI(1) hereafter), real-valued $\mathrm{AR}(1)$ process $\left\{S_{t}\right\}$

$$
\Delta S_{t}=-\frac{\alpha}{T} S_{t-1}+\varepsilon_{t}, \alpha \geq 0, S_{0} \stackrel{\text { a.s. }}{=} 0
$$

and a stochastic process $\left\{X_{t}\right\}$ which is obtained by properly mapping the sample paths of $\left\{S_{t}\right\}$ onto an interval $[\underline{b}, \bar{b}]$. This can be done by requiring that $\left\{\Delta X_{t}\right\}$ depends on $\left\{\Delta S_{t}\right\}$ in such a way that

$$
X_{t} \in[\underline{b}, \bar{b}] \text {, all } t
$$

almost surely (that is, $\Delta X_{t} \in\left[\underline{b}-X_{t-1}, \bar{b}-X_{t-1}\right]$ a.s., all $t$ ). The simplest process satisfying these requirements is obtained by assuming that, conditionally on $X_{t-1}, \Delta X_{t}$ is determined by truncating $\Delta S_{t}$ between $\underline{b}-X_{t-1}$ and $\bar{b}-X_{t-1}$; alternatively, by censoring $\Delta S_{t}$ in $\underline{b}-X_{t-1}$ and $\bar{b}-X_{t-1}$. Both (censored and truncated) types of behavior near the limits can be nested within a more general class of limited processes. The conditions which define such a class are given as follows, where ' $\stackrel{w}{\rightarrow}$ ' and ' $\stackrel{p}{\rightarrow}$ ' denote weak convergence and convergence in probability respectively.

Definition 1 A stochastic process $\left\{X_{t}\right\}_{0}^{T}$ is called 'limited near-integrated of order 1', or 'bounded near-integrated of order 1', briefly $\mathrm{BNI}(1)$, if it satisfies (2) and the following set of conditions:

A1. process $u_{t}:=\Delta X_{t}$ can be decomposed as $u_{t}=\Delta S_{t}+\underline{\xi}_{t}-\bar{\xi}_{t}$, where $\left\{S_{t}\right\}$ satisfies (1) and $\left\{\underline{\xi}_{t}\right\},\left\{\bar{\xi}_{t}\right\}$ are non-negative processes such that, 
conditionally on $\left(X_{t-1}, \Delta S_{t}\right), \underline{\xi}_{t}>0$ iff $X_{t-1}+\Delta S_{t}<\underline{b}$ and $\bar{\xi}_{t}>0$ iff $X_{t-1}+\Delta S_{t}>\bar{b}$;

A2. $\left\{\varepsilon_{t}\right\}$, see (1), is a zero-mean process satisfying the invariance principle $T^{-1 / 2} \sum_{t=1}^{[\cdot T]} \varepsilon_{t} \stackrel{w}{\rightarrow} \lambda B(\cdot)$, where $\lambda>0$ and $B(\cdot)$ is a standard Brownian motion on $C[0,1]$; moreover, $(1 / T) \sum_{t=1}^{T} \varepsilon_{t}^{2} \stackrel{p}{\rightarrow} \sigma^{2}, 0<$ $\sigma^{2}<\infty$, and $\max _{t=1, \ldots, T}\left|\varepsilon_{t}\right|$ is $o_{p}\left(T^{1 / 2}\right)$;

A3. $\max _{t=1, \ldots, T} \underline{\xi}_{t}=o_{p}\left(T^{1 / 2}\right)$ and $\max _{t=1, \ldots, T} \bar{\xi}_{t}=o_{p}\left(T^{1 / 2}\right)$;

A4. $\underline{b}=\underline{c} \lambda T^{1 / 2}, \bar{b}=\bar{c} \lambda T^{1 / 2}, \underline{c}<\bar{c} ; X_{0}=c_{0} \lambda T^{1 / 2}, \underline{c} \leq c_{0} \leq \bar{c}$.

When $\alpha=0,\left\{X_{t}\right\}$ is called 'limited integrated of order 1 ', or 'bounded integrated of order 1', briefly $\mathrm{BI}(1)$.

\section{REMARKS}

1. Condition $\mathcal{A}:=\{A 1, A 2, A 3, A 4\}$ defines the time series behavior of $\left\{X_{t}\right\}$. The basic principle is to separate the effect of the boundaries from the dynamics which characterize $\left\{\Delta X_{t}\right\}$ in the absence of range restrictions. This is achieved through the decomposition of error process $\left\{u_{t}\right\}$ given in condition $A 1$. Specifically, $A 1$ implies that $\left\{X_{t}\right\}$ has the component representation $\Delta X_{t}=\Delta S_{t}+\underline{\xi}_{t}-\bar{\xi}_{t}$, and hence $X_{t}=X_{0}+S_{t}-M_{t}, M_{t}:=\sum_{i=0}^{t-1}\left(\underline{\xi}_{t-i}-\bar{\xi}_{t-i}\right)$. Since in the absence of limits $X_{t}=X_{0}+S_{t}$, the difference $M_{t}=\left(X_{t}-X_{0}\right)-S_{t}$ represents the (cumulated) amount which controls the trajectory of $\left\{X_{t}\right\}$ in order to satisfy the range constraint (2). Note that $\underline{\xi}_{t}$ and $\bar{\xi}_{t}$ are different from zero if and only if $X_{t-1}+\Delta S_{t}$ does not respect the range constraint. When $X_{t-1}+\Delta S_{t}>\bar{b}$ (or $X_{t-1}+\Delta S_{t}<\underline{b}$ ), $\bar{\xi}_{t}$ $\left(\underline{\xi}_{t}\right)$ is large enough to ensure that $X_{t}$ satisfies $(2) .^{2}$

\footnotetext{
${ }^{2}$ The rationale behind Assumption $\mathcal{A}$ is that any truncated/censored/reflected random variable (r.v.) can be written as a random transformation of a r.v. with infinite support. Consider for ease of notation the lower limit case only, and suppose that a r.v. $u$ is censored at $\underline{b}$ and that its distribution function (df) has the form $F_{u}(x)=G(x) \mathbb{I}(x \geq \underline{b})$, where $G(\cdot)$ is an uncensored df. The same distribution can be obtained by taking $u:=\varepsilon+\underline{\xi}$, where $\varepsilon$ is a r.v. with df $G(\cdot)$ on all the real set and $\underline{\xi}:=(\underline{b}-\varepsilon) \mathbb{I}(\varepsilon<\underline{b})$. Truncation at $\underline{b}$, i.e. $F_{u}(x)=$
} 
2. The process $\left\{\varepsilon_{t}\right\}$, see $A 2$, only needs to satisfy an invariance principle and is therefore Rényi-mixing (Phillips and Ouliaris, 1990), or I(0) in a broad sense. Hence, the local-to-unity asymptotics of Phillips (1987b) imply that $\left(\lambda^{2} T\right)^{-1 / 2} S_{[\cdot T]} \stackrel{w}{\rightarrow} J_{\alpha}(s):=\int_{0}^{s} \exp \{-\alpha(s-r)\} d B(r)$, i.e. an Ornstein-Uhlenbeck process. For $\alpha>0,\left\{S_{t}\right\}$ is therefore nearintegrated. In the special case $\alpha=0, \Delta S_{t}=\varepsilon_{t}$ and $\left\{S_{t}\right\}$ is I(1). Note that $\left\{\varepsilon_{t}\right\}$ should also satisfy a milder regularity condition, i.e. that the extreme process $\max _{t=1, \ldots, T}\left|\varepsilon_{t}\right|$ does not diverge 'too much' and that a second-order weak law of large numbers applies.

3. Condition $A 3$ is a technical assumption which is needed in order to avoid that $\left\{X_{t}\right\}$ 'jumps' at the boundaries. Specifically, $A 2$ and $A 3$ together implies that $\max _{t}\left|\Delta X_{t}\right|$ is $o_{p}\left(T^{1 / 2}\right)$.

4. Condition $A 4$ may appear unusual. It states the relation between the position of the limits $(\underline{b}, \bar{b})$ and the sample size $T$. $A 4$ is a key condition in order to assess both empirically and theoretically to what extent the limits impact the behavior of the process. As it will be stressed later on, $\underline{c}$ and $\bar{c}$ in $A 4$ provide a way to measure the influence of such limits in finite samples. Moreover, they allow to derive an asymptotic theory in the presence of range restrictions without modelling the behavior of the process near the limits in a parametric fashion. Finally, $A 4$ enables us to obtain a convenient unification of the (near-) unit root asymptotic theory with the limited-dependent variable framework, and also to modify standard unit root inference in order to take account of range constraints properly.

5. Since the limit parameters $\underline{b}$ and $\bar{b}$ depend on $T$, a time series generated according to Definition 1 formally constitutes a triangular array of the type $\left\{X_{t, T}: t=0,1, \ldots, T ; T=0,1, \ldots\right\}$, see e.g. Phillips (1987b). This notation is not essential to the discussion below; hence, limited (near-)integrated processes will be simply denoted as $\left\{X_{t}\right\}$.

$[1-G(\underline{b})]^{-1}[G(x)-G(\underline{b})] \mathbb{I}(x \geq \underline{b})$ can be obtained by simply taking $\varepsilon$ as a r.v. with d.f. $G(\cdot)$ and by taking $\underline{\xi}$ as a r.v. such that $F_{\underline{\xi} \mid \varepsilon<\underline{b}}(x)=F_{u}(x+\varepsilon)$ and $F_{\underline{\xi} \mid \varepsilon \geq \underline{b}}(x)=\mathbb{I}(x \geq 0)$. Reflection at $\underline{b}$, i.e. $F_{u}(x)=[G(x)-G(2 \underline{b}-x)] \mathbb{I}(x \geq \underline{b})$ is given by taking $\varepsilon$ with d.f. $G(\cdot)$ and defining $\underline{\xi}:=(2 \underline{b}-x) \mathbb{I}(\varepsilon<\underline{b})$. 
6. A bounded near-integrated process reverts (i) because of the limits $[\underline{b}, \bar{b}]$ and, if $\alpha>0$ in equation (1), (ii) because its driving process $\left\{S_{t}\right\}$ has no unit roots. In the special case of bounded integrated dynamics, i.e. $\alpha=0$, the process is mean reverting in the close neighborhood of $\underline{b}$ and $\bar{b}$ only; hence $\mathrm{BI}(1)$ processes differ from standard $\mathrm{I}(1)$ processes just because of the range constraints. In Section 5 it will be shown how the constraint $\alpha=0$ implied by the $\mathrm{BI}(1)$ model can be tested against the alternative hypothesis of limited autoregressive dynamics without a unit root.

A basic result is that limited $\mathrm{NI}(1)$ processes satisfy an invariance principle as in the standard NI(1) framework; in this case, however, the parameters $\underline{c}$ and $\bar{c}$ enter the limit Brownian functional. In order to derive this property, we need to introduce the following definition.

Definition 2 Let $Z$ be a stochastic process in $C[0,1]$. The bivariate process $(L, U)$ is said to be a 'two-sided regulator' of $Z$, with limits $a, b, a<b$, if (i) $Z_{a}^{b}(s):=Z(s)+L(s)-U(s) \in[a, b]$, (ii) $L$ and $U$ are increasing and continuous with $L(0)=U(0)=0$ a.s., (iii) $L$ and $U$ increases only when $Z_{a}^{b}=a$ and $Z_{a}^{b}=b$ respectively. If $Z=B$, i.e. a standard Brownian motion, then $Z_{a}^{b}$ is called 'regulated Brownian motion'.

The two-sided regulator controls the trajectory of a $C[0,1]$ process by keeping its sample paths between the given limits $a, b$; the regulated process lies in $C[0,1]$ as well. The reader can refer to Harrison (1985) and Dixit (1993) for further insights.

Consider the follow continuous-time approximant of $\left\{X_{t}\right\}$

$$
X_{T}(s):=\frac{1}{\lambda \sqrt{T}}\left(X_{[s T]}-X_{0}\right), s \in[0,1]
$$

on the cadlag space $D:=D[0,1]$. If $D[0,1]$ is endowed with the uniform topology, see Billingsley (1968), the next theorem follows in the unit root case $(\alpha=0)$. 
Theorem 1 Let $\left\{X_{t}\right\}$ be a $\mathrm{BNI}(1)$ process, see Definition 1 . Moreover, let $X_{T}(\cdot)$ be defined as in (3). Then, if $\alpha=0$, as $T \uparrow \infty$ $X_{T}(\cdot) \stackrel{w}{\rightarrow} B_{c-c_{0}}^{\bar{c}-c_{0}}(\cdot)$, where $B_{c-c_{0}}^{\bar{c}-c_{0}}(\cdot)$ is a regulated Brownian motion with boundaries at $\underline{c}-c_{0}, \bar{c}-c_{0}$.

This result differs from standard I(1) asymptotics mainly because the limit process is not a standard Brownian motion, as e.g. in Phillips (1987a), but is a regulated Brownian motion. The sample paths of the limit process are therefore bounded between $\underline{c}-c_{0}$ and $\bar{c}-c_{0}$. Theorem 1 obviously nests usual asymptotics since, for $c_{0}-\underline{c}$ and $\bar{c}-c_{0}$ equal to infinity, the standard invariance principle follows.

\section{The 'bounded unit root' distribution}

In this section we will show how the presence of range constraints modifies the asymptotic framework of unit root tests. Specifically, by relying on the weak convergence results of the previous section, the so-called unit root distribution will be generalized to the case of range constraints.

Given a sample $\left\{X_{t}\right\}_{0}^{T}$ drawn from a $\mathrm{BI}(1)$ process, with $X_{0}=0$, let $\widehat{\rho}_{T}$ be the sample first-order autoregressive coefficient, which solves $\widehat{\rho}_{T} \sum X_{t-1}^{2}=\sum X_{t-1} \Delta X_{t}$, where the summations run from $t=1$ to $t=T$. It is well known that if no range constraints are imposed, namely if $\underline{c}=-\infty$ and $\bar{c}=+\infty, \widehat{\rho}_{T}$ has the following asymptotic distribution (see Phillips, 1987a)

$$
T\left(\widehat{\rho}_{T}-1\right) \stackrel{w}{\rightarrow} \frac{B(1)^{2}-\sigma^{2} / \lambda^{2}}{2 \int_{0}^{1} B(s)^{2} d s}
$$

where $\sigma^{2}:=\operatorname{plim}_{T \rightarrow \infty} T^{-1} \sum_{t=1}^{T} \varepsilon_{t}^{2}$ is assumed to exist. In the special case $\lambda^{2}=\sigma^{2}$, the asymptotic distribution (4) is known as the unit root (or Dickey-Fuller) distribution, $Z$ in the following. By referring to Theorem 1, it is straightforward to extend the asymptotics summarized in (4) to the case of range constraints. This generalization is presented in the next theorem. 
Theorem 2 Under the conditions of Theorem 1, as $T \uparrow \infty$

$$
Z_{\rho}:=T\left(\widehat{\rho}_{T}-1\right) \stackrel{w}{\rightarrow} \frac{B_{\underline{c}}^{\bar{c}}(1)^{2}-\sigma^{2} / \lambda^{2}}{2 \int_{0}^{1} B_{\underline{c}}^{\bar{c}}(s)^{2} d s}
$$

where $B_{\underline{c}}^{\bar{c}}(\cdot)$ is a regulated Brownian motion with boundaries at $\underline{c}, \bar{c}$.

For $\lambda^{2}=\sigma^{2}$ the sample autoregressive coefficient is asymptotically distributed as $\left(2 \int B_{\underline{c}}^{\bar{c}}(s)^{2} d s\right)^{-1}\left(B_{\underline{c}}^{\bar{c}}(1)^{2}-1\right)$, which differs from the usual unit root distribution since it is expressed in terms of functionals of a regulated Brownian motion and not of a standard Brownian motion. This distribution is called a 'bounded unit root distribution', with parameters $\underline{c}$ and $\bar{c}$, and denoted as $Z(\underline{c}, \bar{c})$.

Kernel estimates of the probability density function (pdf) associated with the bounded unit root distribution for various values of $\bar{c}=-\underline{c}=: c>0$, i.e. under symmetric limits around the origin, is reported in Figure 1. These are based on 50,000 Monte Carlo (MC) replications where the limit regulated Brownian motion is obtained as $B_{\underline{c}}^{\bar{c}}(s)=\varphi_{\underline{c}}^{\bar{c}}(B(s))$, where $\varphi_{\underline{c}}^{\bar{c}}(\cdot)$ is the reflection function (see Karatzas and Shreve, 1988, p. 97, for $\underline{c}=0$ and $\bar{c}=a$ ) and the Brownian motion $B(\cdot)$ is approximated by its discrete realization from a sample of size 20,000; the rationale behind this algorithm is that $B_{c}^{\bar{c}}(s) \stackrel{d}{=} \varphi_{c}^{\bar{c}}(B(s))$, see Harrison $(1985)^{3}$. For $c$ sufficiently large the $\mathrm{pdf}$ is close to the standard unit root distribution. The presence of limits translates the asymptotic distribution of the autoregressive coefficient toward negative values: the smaller $c$ is, the more the bounded unit root distribution is skewed toward negative values.

The $5 \%$ quantile of the bounded unit root distribution is reported in Figure 2 for various values of $c{ }^{4}$ A selection of $5 \%$ quantiles of the

\footnotetext{
${ }^{3}$ It is worth noting that, although this algorithm allows exact simulatation of the regulated Brownian motion over a discrete grid, simulation of functionals as e.g. $\int_{0}^{1} g\left(B_{\underline{c}}^{\bar{c}}(s)\right) d s$ requires the discretized time increment $1 / T$ to be extremely fine in order to obtain an accurate assessment of the limit distribution, see Asmussen et al. (1995).

${ }^{4}$ Quantiles of the bounded unit root distribution (as well as the quantiles of the various statistics discussed in the paper) have been estimated over a grid of values for $c=0.20,0.21, \ldots, 1.00,1.02, \ldots, 2.00$.
} 
unit root distribution under symmetric limits is also reported in Table 1 , third column $\left(Z_{\rho}\right)$ with $c_{0} / \underline{c}=0$. Again, for $c$ sufficiently large the quantiles correspond to those of the standard unit root distribution. The $5 \%$ quantile tends to $-\infty$ as $c$ approaches 0 .

Several implications can be derived from the results above. First, in the presence of range constraints the large-sample limit of the firstorder autoregressive coefficient is non-standard. With respect to the usual unit root distribution, the limit 'bounded unit root' distribution has two more non-centrality parameters, $\underline{c}$ and $\bar{c}$. Second, the quantiles of the bounded unit root distribution can be extremely different from those of the standard unit root distribution. To which extent the quantiles differ depends (i) on the distance of the boundaries from the initial value of the process (through the parameters $\underline{c}, \bar{c}$ ) and (ii) on the variability of the innovations to $\left\{X_{t}\right\}$ (through the long-run variance $\lambda^{2}$ ). Third, only for limits sufficiently far from the starting value of the process the quantiles of the bounded unit root distribution are well approximated by the quantiles of the standard unit root distribution.

\section{REMARKS}

7 (Initial CONDitions) The derivation of the bounded unit root distribution is based on the condition $X_{0}=0$. If, however, the process starts in $X_{0}:=c_{0} \lambda T^{1 / 2}$, where $c_{0} \in[\underline{c}, \bar{c}]$, the weak convergence (5) still holds with $(\underline{c}, \bar{c})$ replaced by $\left(\underline{c}-c_{0}, \bar{c}-c_{0}\right)$, provided that $\hat{\rho}$ is based on the deviations from the initial value, i.e. on $\left\{X_{t}-X_{0}\right\}$. Note that the $(5 \%)$ quantiles of the unit root distribution are highly sensitive to the presence of asymmetric boundaries (i.e. $-\left(\underline{c}-c_{0}\right) \neq$ $\left.\bar{c}-c_{0}\right)$, see Table 1 , third column $\left(Z_{\rho}\right)$.

8 (ONE-SIDED LIMITS). One-sided limits can be treated as a special case by setting $\bar{c}=+\infty$ (lower limit) or $\underline{c}=-\infty$ (upper limit). By letting $X_{0}=0$ and $\bar{c}=+\infty$ the $5 \%$ quantile of the bounded unit root distribution $Z(\underline{c},+\infty)$ is reported in Figure 3, while a selection of $5 \%$ quantiles is reported in Table 2 , second column $\left(Z_{\rho}\right)$. It is interesting to observe that, as $\underline{c} \uparrow 0$, i.e. the process starts at the lower limit, the quantiles converge to those of the standard unit root distribution. 
This follows from the distribution equality $B_{0}^{+\infty}(\cdot) \stackrel{d}{=}|B(\cdot)|$, see Harrison (1985, p. xii), which implies that $Z(0,+\infty) \stackrel{d}{=} Z$.

9 (DETERMinistic TERM CORRECTIONS). If the computation of the sample first-order autoregressive coefficient $\widehat{\rho}$ is based on the demeaned series $\left\{X_{t}-\bar{X}\right\}$, the bounded unit root distribution has the same form as derived above, but with $B_{\underline{c}}^{\bar{c}}(\cdot)$ replaced by a demeaned regulated Brownian motion, namely $B_{\underline{c}}^{\overline{\bar{c}}}(\cdot)-\int_{0}^{1} B_{\underline{c}}^{\bar{c}}(s) d s$. On the other hand, the $Z_{\rho}$ statistic calculated from GLS-demeaned data, see Elliott et at. (1996), has the limiting representation given in (5).

In a similar way, if the computation of $\widehat{\rho}$ involves fitting a linear time trend by OLS the limit distribution depends on a demeaned and detrended regulated Brownian motion, namely

$$
B_{\underline{c}}^{\bar{c}}(s)-\widehat{\alpha}-\widehat{\beta} s,(\widehat{\alpha}, \widehat{\beta}):=\arg \min _{\alpha, \beta} \int_{0}^{1}\left(B_{\underline{c}}^{\bar{c}}(s)-\alpha-\beta s\right)^{2} d s .
$$

There are important implications of the convergence results outlined above for unit root testing. Such implications are examined in the next section.

\section{Implications for unit root tests}

The most common approach to testing for a unit root against stable alternatives is to refer to statistic (5) as a left-sided test, i.e. to reject the null of a unit root for large negative values of $Z_{\rho}:=T\left(\widehat{\rho}_{T}-1\right)$. By using the distribution results of the previous section it can be reasonably argued that in some cases the rejection rate of the unit root test can be substantially affected by range constraints.

To stress this result, assume that the d.g.p. is $\mathrm{BI}(1)$ with uncorrelated homoskedastic innovations $\left(\sigma^{2}=\lambda^{2}\right)$. The rejection probability of the $\mathrm{I}(1)$ hypothesis when standard critical values are employed estimated through MC simulation, see the previous section - is reported in Figure 4; the significance level is set at 5\%. As expected, 
the rejection frequency is strongly related to the position of the limits. There are at least two important consequences deriving from this result.

On the one hand, since standard unit root inference in the framework of limited time series could point to the rejection of the unit root hypothesis, the researcher might erroneously conclude that the process has no unit root whereas the d.g.p. is actually $\mathrm{I}(1)$ but subject to (one-sided or two-sided) range constraints. When testing for unit roots in the presence of limited time series, an analysis of the 'negligibility' of the limits is therefore a necessary step before interpreting the outcome of standard unit root tests in the usual way. Such a step is usually missing when unit root techniques are applied to the analysis of limited time series.

On the other hand, Figure 4 also explains why the practitioner might fail to reject the (false) standard I(1) model in the presence of boundaries. As far as the boundaries are sufficiently far away, the rejection frequencies of the unit root test when the d.g.p. is $\mathrm{I}(1)$ or $\mathrm{BI}(1)$ are in fact identical. That is, unit root tests are not able to detect the presence of the limits.

A further important implication is that, despite the fact that the bounded unit root distribution (as well as the asymptotic distributions of other unit root test statistics, see below) depends on three nuisance parameters, namely $\left(\underline{c}-c_{0}, \bar{c}-c_{0}, \sigma / \lambda\right)$, the unit root statistic (5) can be rearranged in order to eliminate $\sigma / \lambda$ from its asymptotic distribution; this can be achieved by following Phillips' (1987a) approach. Let $\left(\widehat{\lambda}^{2}, \widehat{\sigma}^{2}\right)$ be two consistent estimators for $\left(\lambda^{2}, \sigma^{2}\right)$ in the absence of range constraints (i.e. when $\bar{c}=+\infty$ and $\underline{c}=-\infty$ ), see Davidson and de Jong (2000) and references therein; $\widehat{\lambda}^{2}$ is assumed to be a sum-of-covariances estimator and can be based both on first-differenced data and on first-order autoregression residuals. The following lemma holds under some mild regularity conditions (details are reported in the Appendix).

Lemma 3 Let the conditions of Theorem 1 hold. Then, as $T \uparrow \infty$, $\widehat{\lambda}^{2} \stackrel{p}{\rightarrow} \lambda^{2}$ and $\widehat{\sigma}^{2} \stackrel{p}{\rightarrow} \sigma^{2}$. 
The main consequence of Lemma 3 is that Phillips' (1987a) unit root statistic

$$
\widehat{Z}_{\rho}:=T\left(\widehat{\rho}_{T}-1\right)-\frac{\frac{1}{2}\left(\widehat{\lambda}^{2}-\widehat{\sigma}^{2}\right)}{T^{-2} \sum_{t=1}^{T} X_{t-1}^{2}}
$$

satisfies $\widehat{Z}_{\rho} \stackrel{w}{\rightarrow} Z(\underline{c}, \bar{c})$, i.e. a bounded unit root distribution with parameters $(\underline{c}, \bar{c})$. Hence, the presence of range constraints does not affect the consistency of the estimators of the nuisance parameters $\left(\sigma^{2}, \lambda^{2}\right)$ which do not enter the asymptotic distribution of the unit root statistic. However, $\underline{c}$ and $\bar{c}$ are still two non-centrality parameters affecting the asymptotic distribution of $\widehat{Z}_{\rho}$ and consequently the outcome of the test.

\section{REMARKS}

10 (ONE-SIDED LIMITS). Consider the one-sided limit case, see Remark 8 above. Such a case arises e.g. in the (co-)integration analysis of nominal interest rates. For $X_{0}=0$ and $\bar{c}=+\infty$ the rejection frequencies of the $Z_{\rho}\left(\widehat{Z}_{\rho}\right)$ unit root test are reported for a $5 \%$ nominal level in Figure 5. As in the two-limit case, the rejection frequency essentially depends on the distance between the starting value of the process and the position of the limit. If, however, such a distance is negligible, i.e. $\underline{c} \approx 0$, the quantiles are identical to those of the standard unit root distribution (see Remark 8); this result does not apply when the test involves demeaning (or detrending) the original time series. In general, in the one-limit case the rejection rate is not as high as in the two-limit case; nevertheless, it can considerably exceed the significance level.

11 (Other UNIT ROOT TESTS). Comparable evidence affects most of the procedures usually employed to test for unit roots. The $t$ ratio unit root test $\left(Z_{t}\right.$ hereafter) based on the $t$ statistic associated to $\widehat{\pi}$ in the regression equation $\Delta X_{t}=\pi X_{t-1}+$ error, has asymptotic distribution $(\lambda / \sigma)\left(4 \int B_{\underline{c}}^{\bar{c}}(s)^{2} d s\right)^{-1 / 2}\left(B_{c}^{\bar{c}}(s)^{2}-(\sigma / \lambda)^{2}\right)$. By Lemma 3, Phillips' modified $t$ test $\widehat{Z}_{t}:=(\widehat{\sigma} / \hat{\bar{\lambda}}) Z_{t}-\left\{T\left(\widehat{\sigma}^{2}-\right.\right.$ $\left.\left.\widehat{\lambda}^{2}\right) /(2 \widehat{\lambda})\right\}\left(\sum X_{t-1}^{2}\right)^{-1 / 2}$ converges weakly to $\left(4 \int B_{\underline{c}}^{\bar{c}}(s)^{2} d s\right)^{-1 / 2}\left(B_{\underline{c}}^{\bar{c}}(s)^{2}-\right.$ 
1), which differs from the usual asymptotic distribution since $B_{\underline{c}}^{\bar{c}}(\cdot)$ replaces the Brownian motion $B(\cdot)$. The von Neumann ratio test of Sargan and Bhargava (1983), based on the statistic $V N:=\widehat{\lambda}^{2} T^{2} / \sum X_{t}^{2}$, satisfies the convergence $V N^{-1} \stackrel{w}{\rightarrow} \int B_{\underline{c}}^{\bar{c}}(s)^{2} d s$, which implies that the statistic $V N$ tends to assume larger values with respect to the standard limit distribution $\left(\int B(s)^{2} d s\right)^{-1}$. The variance-ratio test, based on $V R(\delta):=(\widehat{\lambda} T)^{-2} \sum_{t-[\delta T]}^{T}\left(X_{t}-X_{t-[\delta T]}\right)^{2}$, satisfies $V R(\delta) \stackrel{w}{\rightarrow}$ $\int_{\delta}^{1}\left(B_{\underline{c}}^{\bar{c}}(s)-B_{\underline{c}}^{\bar{c}}(s-\delta)\right)^{2} d s$, which is closer to 0 than the standard limit distribution $\int_{\delta}^{1}(B(s)-B(s-\delta))^{2} d s$. Asymptotic sizes for a $5 \%$ nominal level are plotted in figures 4 (two-limit case) and 5 (one-limit case). See also Tables 1 and 2 .

12 (Deterministic term Corrections). As noticed for the bounded unit root distribution, see Remark 8, if the initial condition is $X_{0}=$ $c_{0} \lambda T^{1 / 2}$, results do not change provided that the tests are based on $\left\{X_{t}-X_{0}\right\}$ (or on GLS-demeaned data) and $(\underline{c}, \bar{c})$ is replaced by $\left(\underline{c}-c_{0}, \bar{c}-c_{0}\right)$. If the tests are based on OLS-demeaned (demeaned and detrended) variables, the asymptotic distributions of the considered test statistics depend on a demeaned (demeaned and detrended) regulated Brownian motion.

13 (EMPIRICAL ASSESSMENT OF THE IMPACT OF the Limits). Figures 2 and 4 provide a simple tool to understand to what extent in the basic $\mathrm{BI}(1)$ model with symmetric limits the rejection of the unit root hypothesis could depend on the presence of the limits. Figures 3 and 5 can be referred to in the case of a lower (upper) limit; similar pictures can be easily obtained by simulation under any condition on $\left(\underline{c}-c_{0}, \bar{c}-c_{0}\right)$. Note, however, that since $(\underline{c}, \bar{c})$ are generally not known, they should be at least be estimated consistently. Section 5 tackles this issue.

We end this section by briefly investigating the power function of the $Z_{\rho}$ unit root test in the presence of near-integrated dynamics and range constraints. The d.g.p. is therefore $\mathrm{BNI}(1)$ with $\alpha>0$. Note that the $\mathrm{I}(1)$ hypothesis is violated (i) because the d.g.p. has no unit root and (ii) because of the range constraints. We might therefore 
expect the rejection frequency to be higher than in the usual nearintegrated, $\mathrm{NI}(1)$, case.

To explore this issue we need to derive the asymptotic distribution of the unit root tests when the d.g.p. is $\operatorname{BNI}(1)$ with $\alpha>0$. The next result provides the result for the $Z_{\rho}, \widehat{Z}_{\rho}$ statistics.

Theorem 4 Under the conditions of Theorem 1, if $\alpha>0$ then as $T \uparrow \infty$

$$
Z_{\rho}:=T\left(\widehat{\rho}_{T}-1\right) \stackrel{w}{\rightarrow} \frac{J_{\underline{c} . \alpha}^{\bar{c}}(1)^{2}-\sigma^{2} / \lambda^{2}}{2 \int_{0}^{1} J_{\underline{c} . \alpha}^{\bar{c}}(s)^{2} d s}
$$

where $J_{\underline{c} . \alpha}^{\bar{c}}(s):=J_{\alpha}(s)+L(s)-U(s), J_{\alpha}(\cdot)$ being the diffusion $J_{\alpha}(s)$

$\int_{0}^{s} \exp \{-\alpha(s-r)\} d B(r)$ and $\{L, U\}$ is a two-sided regulator of $J_{\alpha}(\cdot)$ with limits $\underline{c}, \bar{c}$. The heteroskedasticity and autocorrelation robust statistic $\widehat{Z}_{\rho}$ satisfies (6) with $\sigma^{2} / \lambda^{2}$ replaced by unity.

Hence, with respect to the near-integrated framework, the asymptotic distribution of $Z_{\rho}$ depends on a regulated Ornstein-Uhlenbeck process. For $\sigma^{2}=\lambda^{2}$ the distribution (6) can be denoted 'bounded near-unit root distribution', $Z_{\alpha}(\underline{c}, \bar{c})$ hereafter. The theorem can be easily extended to the various unit root tests and to the case of (OLS or GLS) deterministics correction.

The asymptotic rejection frequency of the $Z_{\rho}\left(\widehat{Z}_{\rho}\right)$ test is plotted for various values of $\alpha$ and $c:=\bar{c}=-\underline{c}$ (symmetric case) in Figure 6 . The figures are based on a MC experiment with 50,000 replications where the limit regulated Ornstein-Uhlenbeck process is obtained by applying the two-sided regulator, see Definition 2, to a discrete realization of the Ornstein-Uhlenbeck process over a grid of 20,000 points. In the left panel of the figure, the test is based on deviations from the initial value, while in the right panel it is based on OLS-demeaned data. The ratio $\lambda / \sigma$ is set to unity. For $c=+\infty$ the usual rejection rate of the $Z_{\rho}$ test when the d.g.p. is near-integrated is obtained, see Elliott et al. (1996), Figures 1-2. As expected, Figure 6 shows that in the presence of (symmetric) range constraints the unit root $Z_{\rho}$ test tends to reject more often than in the absence of constraints. 
However, it should be underlined that this result concerns the symmetric case only. When the process starts near the boundaries, the same evidence does not necessarily hold.

\section{Testing the 'bounded unit root' hypothesis}

When there are range constraints, instead of testing the (trivially false) I(1) hypothesis the researcher should be more interested in testing the 'bounded I(1)' hypothesis, against a non-unit root alternative, e.g. the bounded, near-I(1) model. Our framework allows to tackle this testing problem. Specifically, despite the fact that the bounded unit root distribution (as well as the asymptotic distributions of the other unit root test statistics) depends on three nuisance parameters, $\left(\underline{c}-c_{0}, \bar{c}-c_{0}, \sigma / \lambda\right)$, it is possible to define a proper rejection region in order to test the $\mathrm{BI}(1)$ hypothesis at a given significance level, hence avoiding spurious rejections caused by the presence of the range constraints only.

The main result needed to develop a $\mathrm{BI}(1)$ test is given by the following corollary of Lemma 3, which shows that the two unknown parameters $(\underline{c}, \bar{c})$ can be consistently estimated.

Corollary 5 Let the conditions of Theorem 1 hold. Moreover, let $\underline{\widehat{c}}:=\underline{b}\left(\widehat{\lambda}^{2} T\right)^{-1 / 2}$ and $\widehat{\bar{c}}:=\bar{b}\left(\widehat{\lambda}^{2} T\right)^{-1 / 2}$. Then, as $T \uparrow \infty, \underline{\widehat{c}} \stackrel{p}{\rightarrow} \underline{c}$ and $\overline{\bar{c}} \stackrel{p}{\rightarrow} \bar{c}$.

Hence, given that the limits $(\underline{b}, \bar{b})$ are known, from the consistency of $\left(\widehat{\lambda}^{2}, \widehat{\sigma}^{2}\right)$ it follows that the two nuisance parameters of the bounded unit root distribution $(\underline{c}, \bar{c})$ can be consistently estimated by $\underline{\widehat{c}}$ and $\widehat{\bar{c}}$ respectively. Note that $\underline{\hat{c}}$ (and $\widehat{\bar{c}}$ as well) is 'super-consistent', in the following sense: if $T^{\delta}(\widehat{\lambda}-\lambda)$ is $O_{p}(1), \delta>0$, it is straightforward to show that $T^{1 / 2+\delta}(\underline{\widehat{c}}-c)=O_{p}(1)$.

Therefore, if the d.g.p. is a $\mathrm{BI}(1)$ process, the rejection frequency of the unit root test equalizes the selected significance level in large samples as far as the quantiles of the $Z(\underline{\widehat{c}}, \widehat{\bar{c}})$ distribution are used; 
the same result applies to all unit root tests previously discussed ${ }^{5}$. In the following it will be shown that the asymptotics obtained so far provide excellent results in small samples as well. However, the power properties of the $\mathrm{BI}(1)$ test will be investigated beforehand.

\subsection{Asymptotic power}

It is interesting to compare the asymptotic power of $\mathrm{BI}(1)$ tests with the asymptotic power function of standard unit root tests in the absence of range constraints. To analyze this issue we do not need any further theoretical result since the distribution of the unit root statistics under BNI(1) dynamics has already been obtained in Section 4, Theorem 1 and since Corollary 5 remains valid.

In order to assess to which extent the triple $\{\alpha, \underline{c}, \bar{c}\}$ affects the asymptotic power function of the 'bounded unit root' tests, in Figure 7 the asymptotic power function of the $Z_{\rho}$ test is plotted for various values of $\alpha$ and $c:=\bar{c}=-\underline{c}$ (symmetric case). The ratio $\lambda / \sigma$ is set to unity. In the left panel of the figure the test is applied to deviations from the initial value, while in the right panel the test refers to OLS-demeaned data.

As expected, tests of the $\mathrm{BI}(1)$ hypothesis are less powerful than standard I(1) tests, i.e. in the presence of range constraints it is more difficult than in the usual case of no constraints to assess whether a given series has a unit root. Moreover, the smaller $c$, the lower the power of the test. It is also worth noting that, in the case of symmetric constraints, OLS demeaning reduces the power of the test (for $\alpha$ close to zero the test might have no power against BNI(1) alternatives). The same evidence, however, is not always found when asymmetric (or one-sided) boundaries are considered.

\footnotetext{
${ }^{5} \mathrm{~A}$ selection of $5 \%$ quantiles and a GAUSS program for simulating the asymptotic quantiles of unit root tests for any choice of $(\underline{c}, \bar{c})$ are available from the webpage www2.stat.unibo.it/cavaliere/rconstr/.
} 


\section{$5.2 \quad$ Finite sample size}

In this section we briefly report the outcome of a set of Monte Carlo simulations on the small sample properties of the limit-corrected unit root tests outlined above. Since a key assumption ( $A 4$ in Definition 1) of $\mathrm{BI}(1)$ asymptotics is that the position of the limits depends on $T^{1 / 2}$, one could reasonably argue that the small sample accuracy of the test (at least in terms of size) might be inadequate. In the following it will be shown that this is not the case.

Initially, as the d.g.p. a $\mathrm{BI}(1)$ process $\left\{X_{t}\right\}$ is chosen with $\left\{\varepsilon_{t}\right\}$ being a Gaussian i.i.d. process with zero mean and unit variance. The (conditional) distribution of $\left\{\varepsilon_{t}\right\}$ is reflected at the boundaries, see footnote 2 . Due to space constraint, we consider the symmetric limit case only, i.e. $\bar{c}=-\underline{c}=c$ and $X_{0}=0$. Results for the asymmetric case (as well as for different truncation mechanisms) do not substantially differ. We consider the $Z_{\rho}$ and $Z_{t}$ unit root tests, both based on the deviations of the observed series from the initial value and from the sample average, as well as the tests based on autocorrelation and heteroskedasticity corrections, namely the $\widehat{Z}_{\rho}$ and $\widehat{Z}_{t}$ Phillips-Perron tests. For the latter tests, the estimator of the long-run variance $\lambda^{2}$ is based on a quadratic spectral kernel with bandwidth parameter chosen according to Andrews' (1991) automatic data-dependent procedure using the plug-in method based on an $\mathrm{AR}(1)$ model fit to the data. The unit root tests employ the estimator of $c$ defined in Corollary 5, namely $\widehat{c}=\underline{b}\left(\widehat{\lambda}^{2} T\right)^{-1 / 2}=\bar{b}\left(\widehat{\lambda}^{2} T\right)^{-1 / 2}$. The critical values of the asymptotic distribution under the null hypothesis are then retrieved through a linear interpolation of the critical values obtained by simulation in Sections 3 and 4 (recall that the critical values had been simulated for $c$ spanning from $c=0.2$ to $c=2$, with steps 0.01 for $c \leq 1$ and 0.02 for $c>1$ ).

The selected sample sizes are $T=50,100,200,500$ and the number of MC replications is 50,000. Results are summarized in Table 3 . The small sample performance of the tests is excellent. The empirical rejection frequency differs from the significance level only for $c=0.3$ and $T=50$. Nevertheless, when $c=0.3$ and $T$ is set to 100 the 
rejection rate is already very close to the nominal size for most tests. For the other values of $(c, T)$ the asymptotic approximation of the distribution of the tests considered is extremely well-behaving. Tests based on demeaned data are generally better performing in terms of size than tests based on deviations from the initial values. Moreover, $Z_{t}$-type tests are (to some extent) preferable to $Z_{\rho}$-type tests. Finally, the results do not differ when the Phillips-Perron correction for autocorrelation is employed.

Table 4 concerns the case of $\operatorname{AR}(1)$ errors; specifically, $\varepsilon_{t}=$ $\phi \varepsilon_{t-1}+v_{t}$ where $v_{t}$ is drawn from i.i.d. $N(0,(1-\phi))$ and $\phi=0.3$. The long-run variance of $\left\{\varepsilon_{t}\right\}$ is therefore equal to unity. In the autocorrelated case the issue of precise estimation of the long-run variance becomes crucial. Hence, together with the Phillips-Perron tests based on Andrews' (1991) HAC estimator of $\lambda^{2}$, the results for unit root tests based on AR-type estimators of $\lambda^{2}$, hereafter $\widehat{\lambda}_{A R}^{2}$, are reported in the following. The modified Phillips-Perron coefficient test is indicated with $\widehat{Z}_{\rho}^{A R}$, see $\mathrm{Ng}$ and Perron (2001, $M Z_{\alpha}$ in their notation), while $\widehat{Z}_{t}^{A R}$ denotes the Said-Dickey-Fuller $t$ test. The number of lags is chosen according to both the BIC and the MAIC criteria defined in Ng and Perron (2001). Only demeaned-based tests are presented in the table.

The simulation evidence is comparable to the one obtained in the white noise case, although the tests tend to be slightly conservative with respect to the usual unbounded I(1) framework. Tests based on $t$-statistics usually perform better than coefficient-based tests, in particular when AR-type estimators of the long-run variance are employed. In the latter case, the AR-BIC criterion is generally preferable to both the AR-MAIC and Andrews' estimator, exactly as in the case of no limits.

Overall, the small sample performance of the asymptotic approximation combined with the use of empirical estimates of the boundary parameters is adequate and the small sample size of the $\mathrm{BI}(1)$ test appears to be largely satisfactory. 


\section{Empirical illustrations}

In this section, unit root inference in the presence of range constraints will be discussed by referring to two common applications of standard I(1) tests to limited time series, namely testing for exchange rate mean reversion in the presence of a target zone and testing for a unit root in the unemployment rate.

\subsection{EMS Target zone exchange rates}

This section examines an empirical problem which has often been tackled in the literature, i.e. testing for exchange rate mean reversion in the presence of a target zone. The reader can refer to Svensson (1993) and Anthony and MacDonald (1998). Economic theories of target zone exchange rates usually associate the rejection of the unit root hypothesis with the presence of intramarginal Central Bank interventions or mean reverting fundamentals (Delgado and Dumas, 1992). However, as noticed by Svensson (1993), the presence of the target zone can be the source of mean reversion of the exchange rate. In this framework, we will briefly show how the outcome of unit root tests in the presence of (target zone-) range constraints can lead to wrong economic conclusions and how the researcher can properly modify the test procedure for taking the target zone into account.

The exchange rates of four currencies are considered, namely the Danish Krone (DK), the French Franc (FF), the Irish Pound (IP), the Dutch Guilder (NG), all against the Deutsche Mark $(\mathrm{DM})^{6}$, see Figure 8. Exchange rates have been transformed by taking logs and multiplying by 100; the observation frequency is daily. The selected sample starts on 87:01:12 and ends on 93:01:29. During such a period, all the (bilateral) exchange rates considered were bounded within $\pm 2.25 \%$ target zones, which had not been realigned.

\footnotetext{
${ }^{6}$ The data were obtained from Ecu-rates extracted from the BIS database. All exchange rates are spot ecu-rates recorded at a daily central bank telephone conference at $2.30 \mathrm{pm}$ Swiss time. The bilateral exchange rates have been calculated from these Ecu-rates.
} 
In Table 5 the (constant-corrected) Phillips-Perron coefficient and $t$ tests $\left(\widehat{Z}_{\rho . c}\right.$ and $\widehat{Z}_{t . c}$ thereafter $)$ are reported. The truncation lag which enters the long run variance estimator $\hat{\lambda}^{2}$ is chosen by following the automatic data-dependent procedure proposed by Andrews (1991) using the plug-in method based on an AR(1) model fit to the residuals of the auxiliary regression of $\Delta X_{t}$ on $\left(1, X_{t-1}\right)^{\prime}$. The selected significance level is $5 \%$.

According to the standard critical values, no evidence of mean reversion is found for the Danish Krone. Conversely, evidence against the I(1) hypothesis is found for the French Franc, the Irish Pound and the Dutch Guilder.

Does the rejection of the I(1) hypothesis depend on the presence of the target zone alone or should it be interpreted as evidence of mean reverting fundamentals and/or intramarginal intervention? Standard unit root analysis does not provide an answer to this question. However, an answer can be given by employing the BI(1) test introduced in Section 5. In Table 6 the estimates of the BNI(1) model are reported as well as the asymptotic $5 \%$ quantiles associated with $\widehat{Z}_{\rho . c}$ and $\widehat{Z}_{t . c}$ under the 'bounded unit root' null hypothesis. For the $\mathrm{DM} / \mathrm{IP}$ and the DM/NG exchange rates, the tests reject even when boundary-corrected critical values are employed. Therefore we can safely conclude that for these two exchange rates there is evidence of mean reversion which cannot be attributed to the presence of the target zone alone.

The result obtained for the $\mathrm{DM} / \mathrm{FF}$ exchange rate is opposite. Contrary to the results obtained when standard critical values are employed, 'bounded unit root' tests do not lead to the rejection of the null hypothesis: the observed mean reversion can be explained with the presence of the target zone alone.

\subsection{U.S. unemployment rate}

In this section we analyze the monthly U.S. unemployment rate among adult males from January, 1948 through August, 1999. These data have been recently analyzed by Caner and Hansen (2001) by means 
of threshold autoregression methods over a slightly shorter period (1956-1999); the reader can refer to their paper for further details on data definition.

Our first question is whether the $\mathrm{I}(1)$ hypothesis is rejected over the considered sample. By construction, the unemployment rate is bounded and therefore the I(1) specification should not provide an adequate representation of the data. We explore this issue by referring to the Phillips-Perron $\widehat{Z}_{t}$ unit root test, based on deviations from the 1948:01 rate as well as on OLS-demeaned data. The longrun variance is estimated through Andrews' (1991) quadratic spectral kernel HAC estimator based on first-order autoregression residuals. The bandwidth parameter is set to 12 in order to take account of the seasonal autocorrelation which affects changes in the unemployment rate. For space constraints we do not consider other unit root tests, whose conclusions do not differ from those summarized here.

The unit root $t$ statistics are given by $\widehat{Z}_{t}=-2.023$ when deviations from the initial value are considered and $\widehat{Z}_{t . c}=-2.962$ on OLS-demeaned data. Both tests reject the I(1) model at the $5 \%$ significance level (critical values are -1.95 and -2.86 respectively). The researcher should therefore investigate whether such a rejection depends on the existence of an upper limit and a lower limit or if it can be attributed to the presence of mean reversion between the limits.

In Table 7 the parameter estimates of the BNI(1) model are reported. The estimates of $\bar{c}-c_{0}$ (above 10) and $\underline{c}-c_{0}$ (around -0.325 ) show that while the upper barrier $(\bar{b}=100)$ is negligible, the lower barrier $(\underline{b}=0)$ is relevant: according to Figure 5 , with a lower barrier at -0.325 the asymptotic rejection frequency of the (5\% nominal level) $\widehat{Z}_{t}$ test is around 0.285 ( 0.138 for the OLS-demeaning test). Therefore, the outcome of unit root tests is likely to be affected by the presence of a lower boundary.

Boundary-corrected critical values can be easily obtained by referring to the criteria outlined in Section 5 . The estimates of the critical values for testing the $\mathrm{BI}(1)$ hypothesis against the $\mathrm{BNI}(1)$ alternative are presented in the last column of Table 7 : when the $\widehat{Z}_{t}$ test is computed on the deviations from the initial value the $5 \%$ critical value 
is -2.800 ; when demeaned data are used the critical value becomes -3.420 . Hence, the $\mathrm{BI}(1)$ hypothesis is not rejected at the $5 \%$ level and the unit root hypothesis should be therefore maintained.

It can be argued that the actual lower barrier which should be taken into account is the 'natural' unemployment rate. If such a rate is set e.g. to 1.9 , the estimated $\mathrm{BI}(1) 5 \%$ critical values become -2.99 (deviations from $X_{0}$ ) and -3.58 (demeaned data), which again leads to the maintenance of the $\mathrm{BI}(1)$ hypothesis.

Finally, if there is uncertainty on the position of the actual lower (or upper) boundary, instead of attempting to estimate $\underline{c}-c_{0}$, see above, a conservative test for the $\mathrm{BI}(1)$ hypothesis can be constructed. Such a test is obtained by taking the largest (negative) critical value over all admissible values of $\underline{c}-c_{0}$. In the case of the unemployment rate, which is non-negative, $\underline{c}-c_{0}$ must belong to the set $\left[-X_{0} /\left(\lambda T^{1 / 2}\right), \min _{t \leq T}\left\{\left(X_{t}-X_{0}\right) /\left(\lambda T^{1 / 2}\right)\right\}\right]$, where the unknown parameter $\lambda$ has to be replaced with its consistent estimator $\hat{\lambda}$. The obtained (conservative) $5 \%$ critical values for the U.S. male unemployment rate are -3.46 (deviations from $X_{0}$ ) and -3.66 (demeaned data), not against the $\mathrm{BI}(1)$ hypothesis.

Overall, the unit root model with range constraints provides a non-linear representation of the U.S. male unemployment rate dynamics. Note that by using a different non-linear model, i.e. Caner and Hansen's (2001) Threshold-AR model, strong evidence of nonlinearity (i.e. threshold effects) is found over the 1948-1999 period but the unit root hypothesis is hardly rejected ${ }^{7}$. The $\mathrm{BI}(1)$ tests obtained in this section provide comparable evidence, although nonlinearity is here explained by the impact of a lower boundary while in Caner and Hansen's (2001) model non-linearity depends on lagged changes in the unemployment rate over a given time interval.

\footnotetext{
${ }^{7}$ Caner and Hansen (2001) find stronger evidence against the unit root hypothesis over the 1978:12-1999:09 period, but fail to reject it over the 1956:01-1978:11 period. Mixed evidence is found over the full sample (1956-1999).
} 


\section{Extensions and concluding remarks}

The paper shows how the presence of range constraints affects the asymptotic distribution of unit root tests. Testing for unit roots in limited time series should always be carried out with caution since, when the unit root hypothesis is rejected, range constraints can be the cause of the rejection. The approach suggested in the paper provides a way to assess the role of range constraints and it can be implemented easily. It allows a quick evaluation of the relevance of the limits, and also to test statistically if a given limited time series reverts because of the presence of the limits alone (the 'bounded unit root' hypothesis) or because it is mean reverting within such limits (the 'bounded, nearunit root' hypothesis). The proposed asymptotic framework provides an extremely adequate approximation of the finite sample properties of unit root tests under range constraints.

The results obtained can be well extended to a multivariate framework. While in the univariate case the presence of limits can bias sample autocorrelations (as well as the probability of maintaining the I(1) hypothesis) toward unity, in the multivariate case the limits can increase the degree of cross-correlation between two or more series. That is, the correlation which is spuriously induced by range constraints might be erroneously confused with the existence of systematic covariation. Comparable outcomes can affect multivariate unit root as well as cointegration tests, in that the stronger the range constraints on the data, the higher the probability of the tests to point toward (spurious) cointegration. The asymptotics and the finite-sample methods discussed in this paper can be easily extended to a multivariate framework.

Finally, it is worth noting that the asymptotics obtained provide a basis for (asymptotic) power comparisons when the researcher wants to test whether a given time series with (near-) integrated behavior is bounded by unobservable limits. For example, in the context of floating nominal exchange rates one might be interested in testing whether a given bilateral exchange rate is regulated within an undeclared target zone. The asymptotics of Section 4 show which unit 
root tests are preferable in terms of power when the standard I(1) hypothesis is tested against the bounded I(1) alternative.

\section{A Mathematical Appendix}

Proof of Theorem 1. Consider the process $\left\{\widetilde{X}_{t}\right\}$ defined recursively as

$$
\widetilde{X}_{t}=\left\{\begin{array}{cc}
\widetilde{X}_{t-1}+\varepsilon_{t} & \text { if } \widetilde{X}_{t-1}+\varepsilon_{t} \in[\underline{b}, \bar{b}] \\
\bar{b} & \text { if } \widetilde{X}_{t-1}+\varepsilon_{t}>\bar{b} \\
\underline{b} & \text { if } \widetilde{X}_{t-1}+\varepsilon_{t}<\underline{b}
\end{array}\right.
$$

with initial condition $\widetilde{X}_{0}=X_{0}$. By setting $\widetilde{X}_{T}(\cdot):=\left(\lambda^{2} T\right)^{-1 / 2} \widetilde{X}_{[\cdot T]}$ the following Theorem holds.

Theorem 6 Under the conditions of Theorem 1, as $T \uparrow \infty \widetilde{X}_{T}(\cdot) \stackrel{w}{\rightarrow}$ $B_{\underline{c}-c_{0}}^{\bar{c}-c_{0}}(\cdot)$, where $B_{\underline{c}-c_{0}}^{\bar{c}-c_{0}}(\cdot)$ is a regulated Brownian motion with boundaries at $\underline{c}-c_{0}, \bar{c}-c_{0}$.

To complete the proof it is sufficient to refer to the following Lemma.

Lemma $7\left\{X_{t}\right\}$ and $\left\{\widetilde{X}_{t}\right\}$ satisfy the condition

$$
\max _{t=0, \ldots, T}\left|X_{t}-\widetilde{X}_{t}\right| \leq \max \left(\max _{t=0, \ldots, T} \bar{\xi}_{t}, \max _{t=0, \ldots, T} \underline{\xi}_{t}\right) \leq \max _{t=0, \ldots, T} \bar{\xi}_{t}+\max _{t=0, \ldots, T} \underline{\xi}_{t}
$$

Since Lemma 7 and condition $A 3$ imply that $\sup _{s}\left|X_{T}(s)-\widetilde{X}_{T}(s)\right| \stackrel{p}{\rightarrow}$ 0 we can apply Billingsley (1968), Theorem 4.1, to conclude that Theorem 6 holds for $X_{T}(\cdot)$ as well.

Proof of Theorem 6 . The proof consists of two steps. First, in the $C[0,1]$ space endowed with the uniform metric, we define a continuous approximant which satisfies Harrison's construction of the regulated Brownian motion, and weak convergence is proved. Then, it is shown that weak convergence holds for the $D[0,1]$ version too. 
To simplify notation set w.l.g. $\underline{c}=0$ and initial value $\widetilde{X}_{0}=$ $c_{0} \lambda T^{1 / 2}, c_{0}>0$, see $A 4$. Process $\widetilde{X}_{t}$ can be recursively defined as $\widetilde{X}_{t}=\widetilde{X}_{0}+S_{t}+L_{t}-V_{t}$, where $S_{t}=\sum_{i=1}^{t} \varepsilon_{i}, L_{t}=\sum_{i=1}^{t} l_{i}$, $V_{t}=\sum_{i=1}^{t} v_{i}, l_{t}=-\left(\widetilde{X}_{t-1}+\varepsilon_{t}\right) \mathbb{I}_{\left\{\widetilde{X}_{t-1}+\varepsilon_{t}<0\right\}}$ and $v_{t}=\left(\widetilde{X}_{t-1}+\varepsilon_{t}-\right.$ $\left.\bar{c} \lambda T^{1 / 2}\right) \mathbb{I}_{\left\{\tilde{X}_{t-1}+\varepsilon_{t}>\bar{c} \lambda T^{1 / 2}\right\}}$. Clearly, $\widetilde{X}_{t} \in\left[0, \bar{c} \lambda T^{1 / 2}\right]$, all $t$. In order to define a $C[0,1]$ approximant of $\widetilde{X}_{t}$, say $\widetilde{X}_{T}(s)$, let us define $C[0,1]$ approximations for all its components, i.e. $S_{t}, L_{t}$ and $V_{t}$. For the partial sum $S_{t}$ we can set

$$
S_{T}(s)=\frac{1}{\lambda \sqrt{T}} \sum_{i=1}^{[s T]} \varepsilon_{i}+\varepsilon_{[s T]+1} \frac{(s T-[s T])}{\lambda \sqrt{T}}
$$

which represents the process obtained by joining the points $\left(t / T,\left(\lambda^{2} T\right)^{-1 / 2} S_{t}\right)$ by means of straight lines. For $V_{t}$ and $L_{t}$ we define this approximation in a slightly different way:

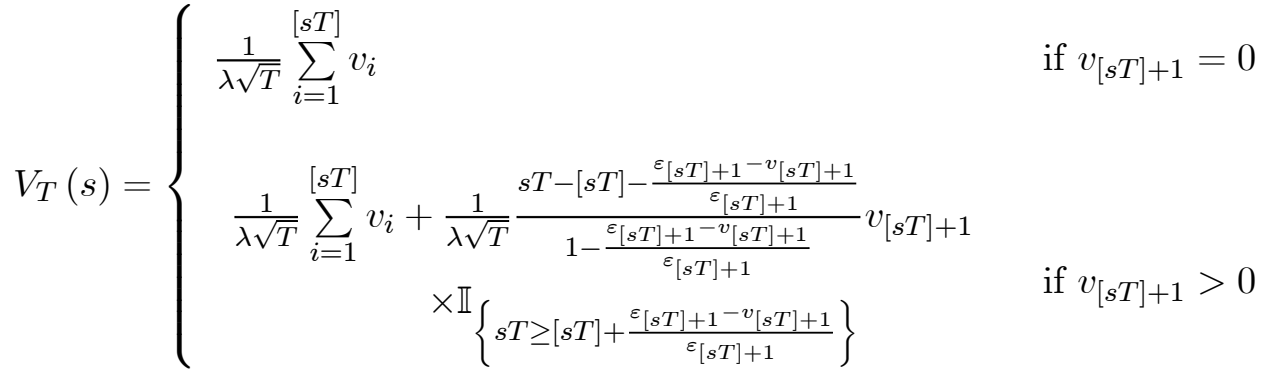

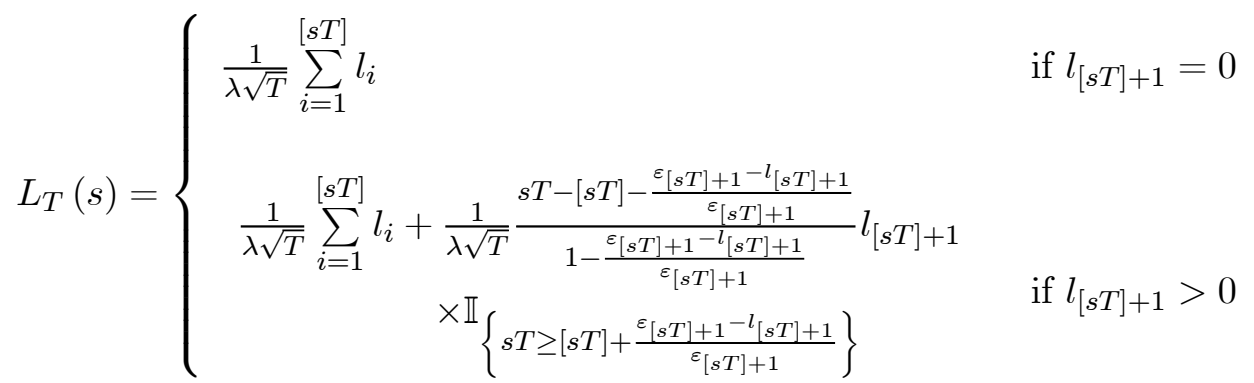

With respect to linear interpolations like (9), this construction still interpolates $\left(\lambda^{2} T\right)^{-1 / 2} X_{t}$ but also satisfies the following properties: 
1. $L_{T}(\cdot)$ and $V_{T}(\cdot)$ are increasing and continuous with $L_{T}(0)=$ $V_{T}(0)=0$;

2. $\widetilde{X}_{T}(s)=c_{0}+S_{T}(s)+L_{T}(s)-V_{T}(s) \in[0, \bar{c}]$, all $s \in[0,1]$;

3. $L_{T}(\cdot)$ and $V_{T}(\cdot)$ increase only when $\widetilde{X}=0$ and $\widetilde{X}=\bar{c}$ respectively.

From Harrison (1985), Proposition 2.4.6, the continuous mapping $\widetilde{X}_{T}(\cdot)=g_{0}^{\bar{c}}\left(S_{T}(\cdot)\right)=S_{T}(\cdot)+c_{0}+L_{T}(\cdot)-V_{T}(\cdot)$ is the unique functional which regulates $S_{T}(\cdot)$ to lie within the interval $[0, \bar{c}]$ and which satisfies properties $1-3$. This allows to obtain the limit distribution of $\widetilde{X}_{T}(\cdot)$ by applying the Continuous Mapping Theorem (CMT, see Billingsley, 1968) to the limit of $S_{T}(\cdot)$. Specifically, $S_{T}(s) \stackrel{w}{\rightarrow} B(s)$ implies that $\widetilde{X}_{T}(s)=g_{0}^{\bar{c}}\left(S_{T}(s)\right) \stackrel{w}{\rightarrow} g_{0}^{\bar{c}}(B(s))$, which is a regulated Brownian motion.

To prove weak convergence on the cadlag space $D[0,1]$ it is sufficient to prove that the process

$$
\begin{aligned}
\widetilde{X}_{T}(s)-\frac{\widetilde{X}_{[s T]}}{\lambda \sqrt{T}}= & \left(L_{T}(s)-L_{[s T]}\right)-\left(V_{T}(s)-V_{[s T]}\right) \\
& +\frac{1}{\lambda \sqrt{T}} \varepsilon_{[s T]+1}(s T-[s T])
\end{aligned}
$$

converges to 0 in probability on $C[0,1]$. Since both $\left|L_{T}(s)-L_{[s T]}\right|$ and $\left|V_{T}(s)-V_{[s T]}\right|$ are smaller than $\left(\lambda^{2} T\right)^{-1 / 2}\left|\varepsilon_{[s T]+1}\right|$, and since the set of increasing points of $L_{T}(s)$ and the set of increasing points of $V_{T}(s)$ are disjoint, it follows that

$$
\left|\tilde{X}_{T}(s)-\frac{\tilde{X}_{[s T]}}{\lambda \sqrt{T}}\right| \leq \frac{2}{\lambda \sqrt{T}}\left|\varepsilon_{[s T]+1}\right|(s T-[s T]) \leq \frac{2}{\lambda \sqrt{T}}\left|\varepsilon_{[s T]+1}\right| ;
$$

therefore

$$
\sup _{s \in[0,1]}\left|\widetilde{X}_{T}(s)-\left(\lambda^{2} T\right)^{-1 / 2} \widetilde{X}_{[s T]}\right| \leq 2\left(\lambda^{2} T\right)^{-1 / 2} \max _{t=1, \ldots, T}\left|\varepsilon_{t}\right| .
$$


By condition $A 2, \max _{t=1, \ldots, T}\left|\varepsilon_{t}\right|$ is $o_{p}\left(T^{1 / 2}\right)$ which implies that (11) is $o_{p}(1)$ and convergence of the process $(10)$ on $D[0,1]$ follows.

Proof of Lemma 7. The lemma is proved by induction. Set $d_{t}=\max _{t^{\prime} \leq t}\left|X_{t}-\widetilde{X}_{t}\right| ;$ since $X_{0}=\widetilde{X}_{0}, d_{0}=0$, and therefore it is enough to show that $d_{t+1} \leq \max \left(\max _{t^{\prime} \leq t+1}\left|\bar{\xi}_{t^{\prime}}\right|, \max _{t^{\prime} \leq t+1}\left|\underline{\xi}_{t^{\prime}}\right|\right)$ given that the relation holds at time $t$. Suppose $X_{t}<\widetilde{X}_{t}$ and $\varepsilon_{t+1}<0$. If $X_{t}+\varepsilon_{t+1}>0,\left|X_{t+1}-\widetilde{X}_{t+1}\right|=\left|X_{t}-\widetilde{X}_{t}\right|, \underline{\xi}_{t+1}=0$ and relation (8) holds. If $X_{t}+\varepsilon_{t+1}<0, X_{t+1}=0$; therefore, if $\widetilde{X}_{t}+\varepsilon_{t+1} \geq$ $0, d_{t+1} \leq d_{t}, \underline{\xi}_{t+1}=0, d_{t+1} \leq \max \left(\max _{t^{\prime} \leq t}\left|\bar{\xi}_{t^{\prime}}\right|, \max _{t^{\prime} \leq t}\left|\underline{\xi}_{t^{\prime}}\right|\right)=$ $\max \left(\max _{t^{\prime} \leq t+1}\left|\bar{\xi}_{t^{\prime}}\right|, \max _{t^{\prime} \leq t+1}\left|\underline{\xi}_{t^{\prime}}\right|\right)$ and the relation holds; on the other side, if $\widetilde{X}_{t}+\varepsilon_{t+1} \leq 0, \widetilde{X}_{t+1}=\underline{\xi}_{t+1}$ and $d_{t+1}=\max \left(d_{t}, \underline{\xi}_{t+1}\right)=$ $\max _{t^{\prime} \leq t}\left(\underline{\xi}_{t+1}\right)$. By similar arguments, the induction proof also holds if $\varepsilon_{t+1} \geq 0$ and symmetrically if $X_{t} \geq \widetilde{X}_{t}$.

Proof of Theorem 2. As in Phillips (1987) write the unit root statistics as the ratio between $(1 / 2)\left(T^{-1} X_{T}^{2}-\widehat{\sigma}^{2}\right), \widehat{\sigma}^{2}:=T^{-1} \sum\left(\Delta X_{t}\right)^{2}$, and $T^{-2} \sum X_{t-1}^{2}$. Regarding the latter term, Theorem 1 and the CMT ensure weak convergence to $\lambda^{2} \int B_{\underline{c}}^{\bar{c}}(s)^{2} d s$; regarding the former term, since $\widehat{\sigma}^{2} \stackrel{p}{\rightarrow} \sigma^{2}$, see Lemma 3 below, from the CMT it holds that $T^{-1} X_{T}^{2}-\widehat{\sigma}^{2} \stackrel{w}{\rightarrow} \lambda^{2}\left(B_{\underline{c}}^{\bar{c}}(1)^{2}-\sigma^{2} / \lambda^{2}\right)$, which completes the proof.

Proof of Lemma 3. We prove the corollary for the case of one limit in 0 and for the estimator

$$
\widehat{\lambda}^{2}=\frac{1}{T} \sum_{j=-T+1}^{T-1} \omega\left(\frac{j}{q_{T}}\right) \sum_{t=|j|+1}^{T} \Delta X_{t} \Delta X_{t-|j|} ;
$$

since under our assumptions the sample first-order autoregressive coefficient satisfies $T(\widehat{\phi}-1)=O_{p}(1)$, see Theorem 2 , results do not change if $\widehat{\lambda}^{2}$ is based on the residuals $X_{t}-\widehat{\phi} X_{t-1}$, i.e. if $\Delta X_{t}$ is replaced by $\left(X_{t}-\widehat{\phi} X_{t-1}\right)$ in (12).

Firstly, the long-run variance estimator can be decomposed as:

$$
\widehat{\lambda}^{2}=\frac{1}{T} \sum_{j=-T+1}^{T-1} \omega\left(\frac{j}{q_{T}}\right) \sum_{t=|j|+1}^{T} \Delta X_{t} \Delta X_{t-|j|}
$$




$$
\begin{aligned}
= & \frac{1}{T} \sum_{j=-T+1}^{T-1} \omega\left(\frac{j}{q_{T}}\right) \sum_{t=|j|+1}^{T}\left(\varepsilon_{t}+\underline{\xi}_{t}\right)\left(\varepsilon_{t-|j|}+\underline{\xi}_{t-|j|}\right) \\
= & \frac{1}{T} \sum_{j=-T+1}^{T-1} \omega\left(\frac{j}{q_{T}}\right) \sum_{t=|j|+1}^{T} \varepsilon_{t} \varepsilon_{t-|j|} \\
& +\frac{1}{T} \sum_{j=-T+1}^{T-1} \omega\left(\frac{j}{q_{T}}\right) \sum_{t=|j|+1}^{T}\left(\underline{\xi}_{t} \varepsilon_{t-|j|}+\varepsilon_{t} \underline{\xi}_{t-|j|}+\underline{\xi}_{t} \underline{\xi}_{t-|j|}\right)(13)
\end{aligned}
$$

where $q_{T}$ is the bandwidth parameter/truncation lag. Assuming that consistency under the no-limit case holds, i.e.

$$
(1 / T) \sum \omega\left(j / q_{T}\right) \sum \varepsilon_{t} \varepsilon_{t-|j|} \stackrel{p}{\rightarrow} \lambda^{2},
$$

to complete the proof we only need to prove that the last term on the r.h.s. of equation (13) goes to 0 in probability. Consider the following inequalities

$$
\begin{aligned}
& \left|\frac{1}{T} \sum_{j=-T+1}^{T-1} \omega\left(\frac{j}{q_{T}}\right) \sum_{t=|j|+1}^{T}\left(\underline{\xi}_{t} \varepsilon_{t-|j|}+\varepsilon_{t} \underline{\xi}_{t-|j|}+\underline{\xi}_{t} \underline{\xi}_{t-|j|}\right)\right| \\
\leq & \frac{1}{T} \sum_{j=-T+1}^{T-1} \omega\left(\frac{j}{q_{T}}\right) \sum_{t=|j|+1}^{T}\left|\underline{\xi}_{t} \varepsilon_{t-|j|}+\varepsilon_{t} \underline{\xi}_{t-|j|}+\underline{\xi}_{t} \underline{\xi}_{t-|j|}\right| \\
\leq & \frac{1}{T} \sum_{j=-T+1}^{T-1} \omega\left(\frac{j}{q_{T}}\right) \max \left(\max _{t=1, \ldots, T}\left|\varepsilon_{t}\right|, \max _{t=1, \ldots, T} \underline{\xi}_{t}\right) \sum_{t=1}^{T} 3 \underline{\xi}_{t} \\
\leq & \frac{3 L_{T}}{T^{1 / 2}}\left(\max _{t=1, \ldots, T} \frac{\left|\varepsilon_{t}\right|}{T^{1 / 2}}+\max _{t=1, \ldots, T} \frac{\underline{\xi}_{t}}{T^{1 / 2}}\right) \sum_{j=-T+1}^{T-1} \omega\left(\frac{j}{q_{T}}\right)
\end{aligned}
$$

as $\underline{\xi}_{t} \geq 0$, all $t$. Note that recursive substitutions allow to express $X_{T}$ as $X_{T}=c_{0} T^{1 / 2}+\sum_{t=1}^{T} \varepsilon_{t}+\sum_{t=1}^{T} \underline{\xi}_{t}$, implying that $T^{-1 / 2} L_{T}=$ $T^{-1 / 2} \sum_{t=1}^{T} \underline{\xi}_{t}=T^{-1 / 2} X_{T}-c_{0}-T^{-1 / 2} \sum_{t=1}^{T} \varepsilon_{t}$ which converges weakly to the well-defined random variable $\lambda\left(B_{-c_{0}}^{+\infty}(1)-B(1)\right)$. Now, suppose that the kernel function $\omega(\cdot)$ satisfies the condition 
$K$. for $\delta \in(0,1 / 2), T^{-\delta} \sum_{j=-T+1}^{T-1} \omega\left(j / q_{T}\right)$ converges to a positive scalar as $T \uparrow \infty$;

and that conditions $A 2$ and $A 3$ are strengthened as follows:

$A 2^{\prime} .\left\{\varepsilon_{t}\right\}$ satisfies $A 2$ and $\max _{t=1, \ldots, T}\left|\varepsilon_{t}\right|=o_{p}\left(T^{1 / 2-\delta}\right)$, where $\delta$ is defined by condition $K$;

$A 3^{\prime} . \max _{t=1, \ldots, T} \underline{\xi}_{t}=o_{p}\left(T^{1 / 2-\delta}\right)$ and $\max _{t=1, \ldots, T} \bar{\xi}_{t}=o_{p}\left(T^{1 / 2-\delta}\right)$, where $\delta$ is defined by condition $K$.

Conditions $K, A 2^{\prime}$ and $A 3^{\prime}$ imply that

$$
\left(\max _{t} T^{-1 / 2}\left|\varepsilon_{t}\right|+\max _{t} T^{-1 / 2} \underline{\xi}_{t}\right) \sum \omega\left(j / q_{T}\right)=o_{p}(1)
$$

and (as $\left.T^{-1 / 2} L_{T}=O_{p}(1)\right)$ that (14) is $o_{p}(1)$ as well.

Finally, by setting $q_{T}=0$ it follows that $\widehat{\sigma}^{2} \stackrel{p}{\rightarrow} \sigma^{2}$. Extension to the two-limit case and to the case of demeaned data is similar. As stressed above, due to the superconsistency of $\widehat{\rho}$ when the d.g.p. is $\mathrm{BI}(1)$ or $\mathrm{BNI}(1)$, see Theorems 2 and 4,the proof continues to hold also if $\widehat{\lambda}^{2}$ is based on first-order autoregression residuals.

Proof of Theorem 4. The proof follows the proof of Theorem 2 with $\varepsilon_{t}$ replaced by $\Delta S_{t}$ and $S_{T} \stackrel{w}{\rightarrow} B$ replaced by $S_{T} \stackrel{w}{\rightarrow} J_{\alpha}$. Note that if the d.g.p. is BNI(1), Lemma 3 continues to hold and hence $\widehat{\lambda}^{2} \stackrel{p}{\rightarrow} \lambda^{2}$. 


\section{References}

Abadir, K.M. and A.M.R. Taylor (1999), On the definition of (co)integration, Journal of time series analysis 20, 129-137.

Andrews, D.W.K. (1991), Heteroskedasticity and autocorrelation consistent covariance matrix estimation, Econometrica 59, 817-58.

Anthony, M. and R. MacDonald (1998), On the mean-reverting properties of target zone exchange rates: some evidence from the ERM, European Economic Review, 42, 1493-1523.

Asmussen, S., P. Glynn and J. Pitman (1995), Discretization error in simulation of one-dimensional reflecting Brownian motion, Annals of Applied Probability 5, 875-896.

Banerjee, A., J. Dolado, J.W. Galbraith and D. Hendry (1993), Cointegration, error-correction, and the econometric analysis of non-stationary data, Oxford: Oxford University Press.

Barr, D.G. and K. Cuthbertson (1991), Neo-classical consumer demand theory and the demand for money, The Economic Journal $101,855-76$.

Billingsley, P. (1968), Convergence of probability measures, New York: John Wiley and Sons.

Blanchard, O.J. and L.H. Summers (1986), in "Isteresis and the European unemployment rate", NBER Macroeconomics Annual, MIT Press, Cambridge, MA.

Caner, M. and B.E. Hansen (2001), Threshold autoregression with a unit root, Econometrica 69, 1555-1596.

Davidson, J. and R. de Jong (2000), Consistency of kernel estimators of heteroskedastic and autocorrelated covariance matrices, Econometrica 68, 407-424. 
Delgado, F. and B. Dumas (1992), Target zones, broad and narrow, in Krugman, P. and M. Miller (eds), Exchange Rate Targets and Currency Bands, Cambridge: Cambridge University Press.

Elliott, G., T.J. Rothemberg and J.H. Stock (1996), Efficient tests for an autoregressive unit root, Econometrica 64, 813-836.

Engle, R.F. and C.W.J. Granger (eds.) (1991), Long run economic relationships - Readings in co-integration, Oxford: Oxford University Press.

Harrison, M.J. (1985), Brownian motion and stochastic flow systems, New York: John Wiley and Sons.

Hsiao C., K. Lahiri, L.F. Lee, M.H. Pesaran (eds.) (1999), Analysis of panels and limited dependent variable models, Cambridge: Cambridge University Press.

Karatzas, I. and S.E. Shreve (1988), Brownian motion and stochastic calculus, New York: Springer-Verlag.

Lindbeck, A. and D.J. Snower (1989), Macroeconomic policy and insider power, American Economic Review 79, 470-376.

Maddala, G.S. (1986), Limited-dependent and qualitative variables in econometrics, Cambridge: Cambridge University Press.

Nelson, C.R. and C.I. Plosser (1982), Trends and random walks in macroeconomic time series, Journal of Monetary Economics 10, $139-162$.

Phillips, P.C.B. (1987a), Time series regression with a unit root, Econometrica 55, 277-301.

Phillips, P.C.B. (1987b) Toward a unified asymptotic theory for autoregression, Biometrika 74, 535-547.

Phillips, P.C.B. (2001), Descriptive econometrics for non-stationary time series with empirical illustrations, Journal of Applied Econometrics 16, 389-413. 
Phillips, P.C.B. and S. Ouliaris (1990), Asymptotic properties of residual based tests for cointegration, Econometrica 58, 165193.

Sargan, J.D. and A. Bhargava (1983), Testing residuals from least squares regression for being generated by the Gaussian random walk, Econometrica 51, 153-174.

Svensson, L.E.O. (1993), Assessing target zone credibility: mean reversion and devaluation expectations in the ERM, 1979-1992, European Economic Review 37, 763-802. 


\begin{tabular}{cccccc}
\hline \hline & \multicolumn{3}{c}{ tests based on $\left\{X_{t}-X_{0}\right\}$} & \multicolumn{2}{c}{ tests based on $\left\{X_{t}-\bar{X}\right\}$} \\
$\bar{c}=-\underline{c}$ & $c_{0} / \underline{c}$ & $Z_{\rho}$ & $Z_{t}$ & $Z_{\rho . c}$ & $Z_{\rho . t}$ \\
\hline 0.3 & 0 & -22.715 & -3.340 & -31.834 & -3.972 \\
& $1 / 4$ & -25.992 & -3.569 & -31.607 & -3.967 \\
& $1 / 2$ & -24.700 & -3.472 & -31.438 & -3.977 \\
& $3 / 4$ & -15.945 & -2.773 & -31.504 & -4.025 \\
0.5 & 1 & -9.093 & -2.082 & -32.339 & -4.136 \\
& 0 & -10.889 & -2.290 & -25.078 & -3.616 \\
& $1 / 4$ & -13.639 & -2.564 & -25.246 & -3.624 \\
& $1 / 2$ & -21.750 & -3.262 & -25.989 & -3.655 \\
& $3 / 4$ & -18.905 & -3.019 & -26.778 & -3.709 \\
0.7 & 1 & -8.106 & -1.946 & -27.971 & -3.857 \\
& 0 & -8.309 & -1.979 & -20.159 & -3.372 \\
& $1 / 4$ & -9.550 & -2.132 & -21.205 & -3.411 \\
& $1 / 2$ & -14.753 & -2.675 & -23.689 & -3.497 \\
& $3 / 4$ & -23.416 & -3.372 & -26.030 & -3.613 \\
0.9 & 1 & -8.106 & -1.946 & -27.822 & -3.808 \\
& 0 & -8.106 & -1.946 & -16.851 & -3.172 \\
& $1 / 4$ & -8.277 & -1.975 & -18.209 & -3.228 \\
& $1 / 2$ & -11.021 & -2.299 & -21.846 & -3.383 \\
& $3 / 4$ & -23.547 & -3.388 & -25.455 & -3.559 \\
1.1 & 1 & -8.106 & -1.946 & -27.822 & -3.793 \\
& 0 & -8.106 & -1.946 & -15.132 & -3.054 \\
& $1 / 4$ & -8.112 & -1.947 & -16.353 & -3.109 \\
& $1 / 2$ & -9.164 & -2.090 & -20.042 & -3.274 \\
& $3 / 4$ & -19.648 & -3.100 & -24.646 & -3.507 \\
& 1 & -8.106 & -1.946 & -27.822 & -3.787 \\
1.5 & 0 & -8.106 & -1.946 & -14.205 & -2.930 \\
& $1 / 4$ & -8.106 & -1.946 & -14.606 & -2.969 \\
& $1 / 2$ & -8.140 & -1.952 & -17.040 & -3.117 \\
& $3 / 4$ & -13.616 & -2.562 & -23.166 & -3.426 \\
& 1 & -8.106 & -1.946 & -27.822 & -3.786 \\
\hline
\end{tabular}

Table 1: Critical values of the Phillips-Perron unit root tests; TWO-SIDED RANGE CONSTRAINTS. NOMINAL LEVEL: $5 \%$.

Notes: When $c_{0} / \underline{c}=0$ the limits are symmetric around the starting value. When $c_{0} / \underline{c}=1$ the process starts on the lower limit. Critical values have been obtained through MC simulation by discretizing the limit regulated Brownian motion over $T=20,000$ segments and using 50,000 replications. 


\begin{tabular}{ccccc}
\hline \hline & \multicolumn{2}{c}{ tests based on $\left\{X_{t}-X_{0}\right\}$} & \multicolumn{2}{c}{ tests based $\left\{X_{t}-\bar{X}\right\}$} \\
$-\underline{c}$ & $Z_{\rho}$ & $Z_{t}$ & $Z_{\rho . c}$ & $Z_{t . c}$ \\
\hline 0.0 & -8.106 & -1.946 & -27.822 & -3.786 \\
0.1 & -16.275 & -2.800 & -26.615 & -3.646 \\
0.2 & -24.177 & -3.431 & -25.813 & -3.575 \\
0.3 & -17.780 & -2.946 & -24.350 & -3.486 \\
0.4 & -12.596 & -2.462 & -22.761 & -3.400 \\
0.5 & -9.965 & -2.182 & -20.974 & -3.314 \\
0.6 & -8.663 & -2.029 & -19.169 & -3.223 \\
0.7 & -8.207 & -1.964 & -17.715 & -3.144 \\
0.8 & -8.112 & -1.948 & -16.525 & -3.087 \\
0.9 & -8.106 & -1.946 & -15.668 & -3.034 \\
1.0 & -8.106 & -1.946 & -15.067 & -2.995 \\
1.2 & -8.106 & -1.946 & -14.448 & -2.945 \\
1.4 & -8.106 & -1.946 & -14.216 & -2.912 \\
1.6 & -8.106 & -1.946 & -14.144 & -2.891 \\
1.8 & -8.106 & -1.946 & -14.139 & -2.878 \\
2.0 & -8.106 & -1.946 & -14.139 & -2.872 \\
2.5 & -8.106 & -1.946 & -14.139 & -2.866 \\
3.0 & -8.106 & -1.946 & -14.139 & -2.864 \\
\hline
\end{tabular}

Table 2: Critical values of the Phillips-Perron unit root tests; ONE-SIDED RANGE CONSTRAINTS. NOMINAL LEVEL: $5 \%$.

Notes: When $\underline{c}=0$ the process starts on the lower limit. Critical values have been obtained through $\mathrm{MC}$ simulation by discretizing the limit regulated Brownian motion over $T=20,000$ segments and using 50,000 replications. 


\begin{tabular}{ccrrrrrrrr}
\hline \hline & \multicolumn{1}{c}{ tests based on $\left\{X_{t}-X_{0}\right\}$} & \multicolumn{3}{c}{ tests based on $\left\{X_{t}-X_{0}\right\}$} \\
$c$ & $T$ & \multicolumn{1}{c}{$Z_{\rho}$} & \multicolumn{1}{c}{$Z_{t}$} & \multicolumn{1}{c}{$\widehat{Z}_{\rho}$} & \multicolumn{1}{c}{$\widehat{Z}_{t}$} & $Z_{\rho . c}$ & $Z_{t . c}$ & $\widehat{Z}_{\rho . c}$ & $\widehat{Z}_{t . c}$ \\
\hline \multirow{2}{*}{0.3} & 50 & 11.875 & 26.615 & 11.785 & 26.625 & 2.135 & 7.325 & 2.345 & 7.860 \\
& 100 & 8.075 & 14.435 & 8.030 & 14.270 & 3.350 & 6.025 & 3.590 & 6.235 \\
& 250 & 5.970 & 7.940 & 5.890 & 7.860 & 3.645 & 4.700 & 3.655 & 4.745 \\
& 500 & 5.320 & 6.345 & 5.230 & 6.240 & 4.075 & 4.515 & 3.885 & 4.395 \\
0.5 & 50 & 5.085 & 7.000 & 5.155 & 7.010 & 3.400 & 6.335 & 3.530 & 6.455 \\
& 100 & 4.870 & 5.660 & 4.885 & 5.685 & 3.775 & 5.320 & 3.980 & 5.455 \\
& 250 & 4.820 & 5.040 & 4.805 & 5.195 & 4.160 & 4.795 & 4.155 & 4.830 \\
& 500 & 4.920 & 5.015 & 4.970 & 5.120 & 4.345 & 4.735 & 4.315 & 4.695 \\
0.7 & 50 & 4.390 & 5.155 & 4.535 & 5.270 & 3.795 & 6.875 & 3.965 & 7.045 \\
& 100 & 4.485 & 4.750 & 4.585 & 4.855 & 4.255 & 5.800 & 4.350 & 5.850 \\
& 250 & 4.545 & 4.740 & 4.595 & 4.785 & 4.640 & 5.355 & 4.635 & 5.390 \\
& 500 & 4.860 & 4.925 & 4.910 & 4.955 & 4.630 & 5.110 & 4.660 & 5.110 \\
0.9 & 50 & 4.330 & 4.955 & 4.500 & 5.100 & 3.685 & 6.670 & 3.975 & 6.940 \\
& 100 & 4.495 & 4.775 & 4.730 & 4.860 & 3.990 & 5.705 & 4.175 & 5.865 \\
& 250 & 4.585 & 4.695 & 4.670 & 4.765 & 4.470 & 5.105 & 4.495 & 5.185 \\
& 500 & 4.815 & 4.885 & 4.885 & 4.975 & 4.635 & 5.015 & 4.780 & 5.130 \\
$\infty$ & 50 & 4.330 & 4.940 & 4.515 & 5.100 & 3.585 & 6.050 & 4.500 & 6.595 \\
& 100 & 4.500 & 4.785 & 4.735 & 4.860 & 4.225 & 5.435 & 4.700 & 5.660 \\
& 250 & 4.580 & 4.690 & 4.665 & 4.755 & 4.365 & 5.135 & 4.750 & 5.340 \\
& 500 & 4.815 & 4.880 & 4.890 & 4.970 & 4.915 & 5.030 & 5.100 & 5.220 \\
\hline
\end{tabular}

Table 3: Finite sample nUll Rejection probabilities WN model; $c=0.3,0.5,0.7,0.9, \infty, T=50,100,250,500$.

Notes: $Z_{\rho}$ and $Z_{t}$ denote the standard Dickey-Fuller coefficient and $t$ tests respectively (without heteroskedasticity and autocorrelation correction). $\widehat{Z}_{\rho}$ and $\widehat{Z}_{t}$ denote the Phillips-Perron autocorrelation and heteroskedasticity-corrected tests. The long-run variance $\lambda^{2}$ is estimated according to Andrews' (1991) HAC estimator with quadratic spectral kernel and $\mathrm{AR}(1)$-automatic bandwidth selection. '.$c$ ' denotes tests based on demeaned data. 


\begin{tabular}{|c|c|c|c|c|c|c|c|}
\hline \multirow[b]{2}{*}{$c$} & \multirow[b]{2}{*}{$T$} & \multicolumn{2}{|c|}{ "Andrews } & \multicolumn{2}{|c|}{$\overline{\text { MAIC }}$} & \multicolumn{2}{|c|}{$\overline{\mathrm{BIC}}$} \\
\hline & & $\widehat{Z}_{\rho}$ & $\widehat{Z}_{t}$ & $\widehat{Z}_{\rho}^{A R}$ & $\widehat{Z}_{t}^{A R}$ & $\widehat{Z}_{\rho}^{A R}$ & $\widehat{Z}_{t}^{A R}$ \\
\hline \multirow[t]{4}{*}{0.3} & 50 & 1.660 & 3.035 & 0.025 & 2.035 & 2.320 & 3.615 \\
\hline & 100 & 1.405 & 2.025 & 0.170 & 1.225 & 5.315 & 1.470 \\
\hline & 250 & 1.565 & 1.765 & 0.710 & 1.280 & 3.085 & 2.090 \\
\hline & 500 & 2.165 & 2.345 & 1.940 & 2.220 & 3.175 & 2.755 \\
\hline \multirow[t]{4}{*}{0.5} & 50 & 2.200 & 4.220 & 0.080 & 3.285 & 2.450 & 5.780 \\
\hline & 100 & 2.100 & 3.080 & 0.140 & 2.360 & 4.965 & 3.615 \\
\hline & 250 & 2.680 & 3.085 & 0.865 & 2.755 & 2.910 & 3.525 \\
\hline & 500 & 2.765 & 3.125 & 1.685 & 3.210 & 2.780 & 3.790 \\
\hline \multirow[t]{4}{*}{0.7} & 50 & 1.760 & 4.580 & 0.185 & 4.085 & 2.540 & 6.850 \\
\hline & 100 & 2.210 & 3.770 & 0.195 & 3.295 & 3.685 & 4.555 \\
\hline & 250 & 2.890 & 3.710 & 0.915 & 3.905 & 2.030 & 4.365 \\
\hline & 500 & 3.460 & 3.905 & 1.385 & 4.120 & 2.005 & 4.640 \\
\hline \multirow[t]{4}{*}{0.9} & 50 & 1.390 & 4.805 & 0.320 & 4.425 & 3.235 & 7.045 \\
\hline & 100 & 2.065 & 3.970 & 0.440 & 3.960 & 4.000 & 5.030 \\
\hline & 250 & 2.900 & 4.110 & 1.400 & 4.330 & 2.300 & 4.680 \\
\hline & 500 & 3.450 & 4.235 & 1.690 & 4.570 & 2.245 & 4.800 \\
\hline \multirow[t]{4}{*}{$\infty$} & 50 & 1.550 & 3.350 & 0.970 & 2.840 & 5.965 & 7.350 \\
\hline & 100 & 2.195 & 3.190 & 1.410 & 3.210 & 7.675 & 5.805 \\
\hline & 250 & 3.205 & 4.055 & 2.770 & 4.380 & 4.625 & 5.165 \\
\hline & 500 & 3.800 & 4.180 & 3.140 & 4.515 & 4.150 & 5.040 \\
\hline
\end{tabular}

Table 4: Finite Sample null Rejection probabilities $A R(1)$ model; $c=0.3,0.5,0.7,0.9, \infty, \phi=0.3, T=50,100,250,500$.

Notes: All tests are based on demeaned data. $\widehat{Z}_{\rho . c}$ and $\widehat{Z}_{t . c}$ denote the PhillipsPerron coefficient and $t$ tests respectively, with long-run variance $\lambda^{2}$ estimated according to Andrews' (1991) HAC estimator with quadratic spectral kernel and $\operatorname{AR}(1)$-automatic bandwidth selection. $\widehat{Z}_{\rho}^{A R}$ and $\widehat{Z}_{t}^{A R}$ denote the Modified coefficient test (Ng and Perron, 2001) and the augumented Said-Dickey-Fuller test respectively. BIC and MAIC denote the critera for determining the number of lags $(k)$ in the Said-Dickey-Fuller regression, see Ng and Perron (2001). When the BIC is used, $0 \leq k \leq 6$ while when the MAIC is used, $0 \leq k \leq\left[12 \cdot(T / 100)^{0.25}\right]$. 


\begin{tabular}{lrrrr}
\hline \hline & \multicolumn{1}{l}{ DK } & \multicolumn{1}{l}{ FF } & \multicolumn{1}{l}{ IP } & \multicolumn{1}{l}{ NG } \\
\hline$\widehat{Z}_{\rho . c}$ & -12.843 & -17.325 & -34.204 & -20.600 \\
$\widehat{Z}_{t . c}$ & -2.648 & -3.243 & -4.009 & -3.242 \\
\hline
\end{tabular}

Table 5: Phillips-Perron unit root tests on EMS eXchange rates, 1987-1993.

Notes: All exchange rates are against the Deutsche Mark. The unit root tests are based on demeaned data. The long-run variance $\lambda^{2}$ is estimated according to Andrews' (1991) HAC estimator with quadratic spectral kernel and AR(1)automatic bandwidth selection. Standard $5 \%$ critical values are -14.10 for the $\widehat{Z}_{\rho . c}$ test and -2.86 for the $\widehat{Z}_{t . c}$ test.

\begin{tabular}{lrrrr}
\hline \hline & \multicolumn{1}{c}{ DK } & \multicolumn{1}{c}{ FF } & \multicolumn{1}{c}{ IP } & \multicolumn{1}{c}{ NG } \\
\hline$\alpha$ & -13.023 & -17.735 & -34.789 & -22.043 \\
$\lambda^{2}$ & 0.013 & 0.010 & 0.012 & 0.001 \\
$\underline{c}-c_{0}$ & -0.402 & -0.320 & -0.342 & -2.466 \\
$\bar{c}-c_{0}$ & 0.617 & 0.804 & 0.711 & 2.102 \\
\hline c.v. $\left(\widehat{Z}_{\text {p.c }}\right)$ & -24.935 & -24.820 & -24.896 & -14.139 \\
c.v. $\left(\widehat{Z}_{t . c}\right)$ & -3.610 & -3.590 & -3.606 & -2.873 \\
\hline
\end{tabular}

Table 6: EMS EXChange Rates: Estimation of the NBI(1) MOdeL AND BOUNDARY-CORRECTED CRITICAL VALUes OF THE PHILLIPS-PERRON UNIT ROOT TESTS.

Notes: Critical values have been obtained through MC simulation by discretizing the limit regulated Brownian motion over $T=20,000$ segments and using 50,000 replications. 


\begin{tabular}{ccccccc}
\hline \hline & $\alpha$ & $\lambda^{2}$ & $\underline{c}-c_{0}$ & $\bar{c}-c_{0}$ & $\widehat{Z}_{t}$ & c.v. $\left(Z_{t}\right)$ \\
\hline $\begin{array}{c}\text { deviations } \\
\text { from } X_{0}\end{array}$ & -3.416 & 0.140 & -0.325 & 10.396 & -2.022 & -2.803 \\
$\begin{array}{c}\text { demeaned } \\
\text { data }\end{array}$ & -7.328 & 0.141 & -0.324 & 10.357 & -2.962 & -3.472 \\
\hline
\end{tabular}

Table 7: U.S. Male Unemployment Rate: Estimation of the NBI(1) MODEL, PHILLIPS-PERRON UNIT ROOT TESTS AND BOUNDARY-CORRECTED CRITICAL VALUES.

Notes: The long-run variance $\lambda^{2}$ is estimated according to Andrews' (1991) HAC estimator with quadratic spectral kernel and AR(1)-automatic bandwidth selection. Standard $5 \%$ critical values are -1.95 for the $\widehat{Z}_{t}$ test and -2.86 for the $\widehat{Z}_{t . c}$ test. Boundary-corrected critical values have been obtained through MC simulation by discretizing the limit regulated Brownian motion over $T=20,000$ segments and using 50,000 replications.

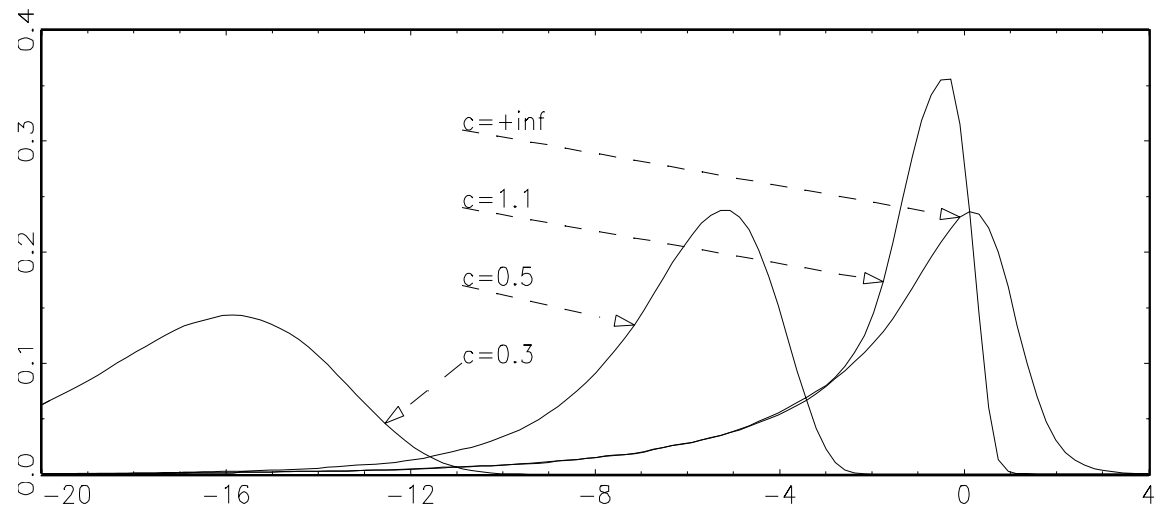

Figure 1: Probability density function of the bounded unit root distribution for $\bar{c}=-\underline{c}=c=0.3,0.5,1.1,+\infty$ (standard unit root distribution). Realizations of the bounded unit root distribution are obtained by discretizing the limit regulated Brownian motion over $T=20,000$ segments and using 50,000 replications; kernel estimates with Epanechnikov weights are computed. 

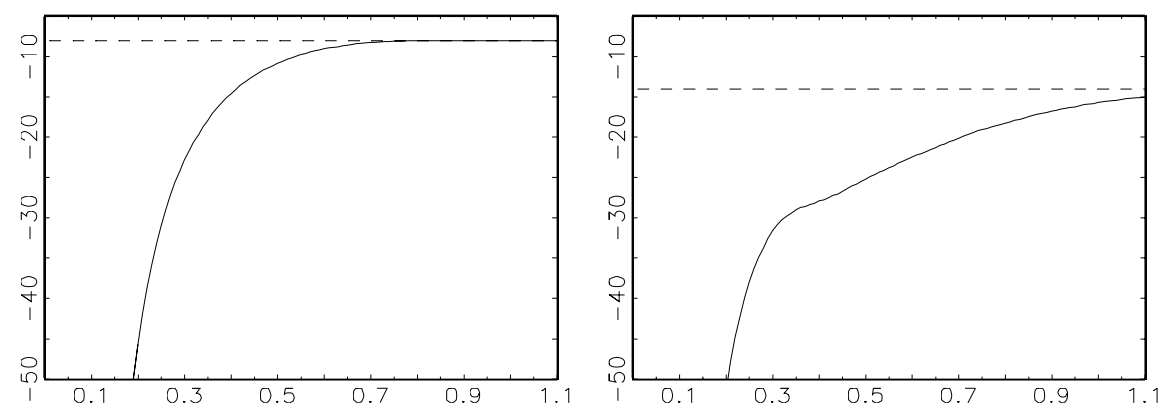

Figure 2: $5 \%$ quantile of the bounded unit root distribution. Left panel: deviations from the initial value. Right panel: demeaned data. Quantiles are obtained by discretizing the limit regulated Brownian motion over $T=$ 20,000 segments and using 50,000 replications.
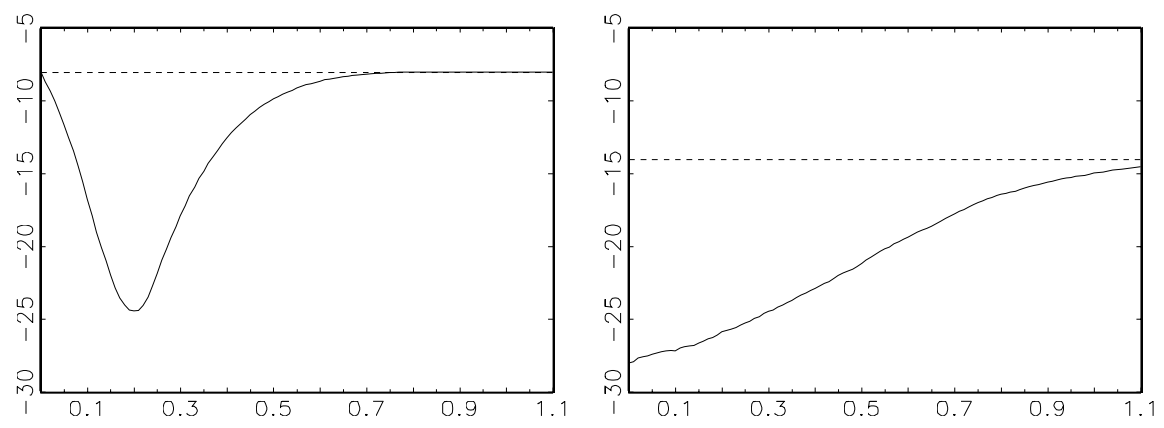

Figure 3: 5\% quantile of the bounded unit root distribution in the one-limit case for various values of $-\underline{c}$. Left panel: deviations from the initial value. Right panel: demeaned data. 

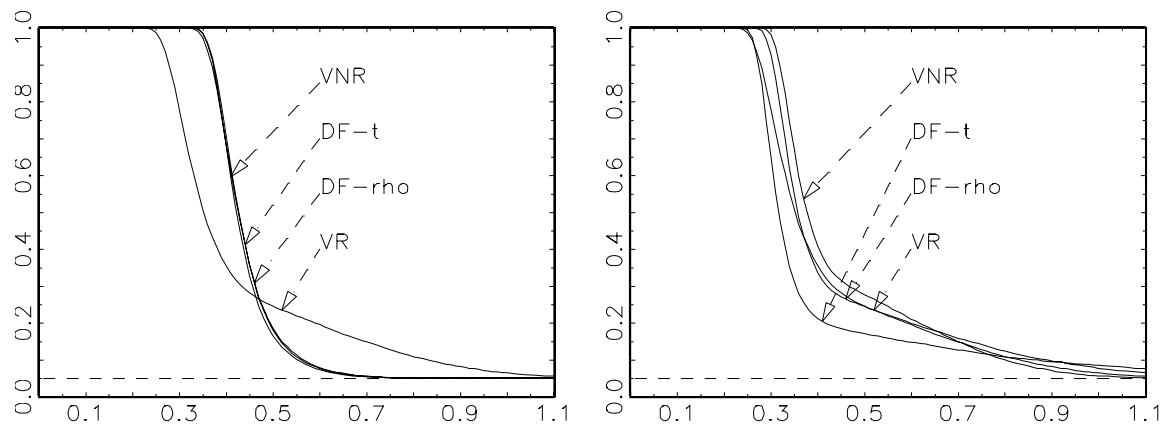

Figure 4: Size of the unit root tests for various values of $\bar{c}=-\underline{c}=c$. DFrho: $Z_{\rho}$ unit root test; DF-t: $Z_{t}$ unit root test; VNR: von Neumann ratio test; VR: variance-ratio test, $\delta=0.5$. Left panel: deviations from the initial value. Right panel: demeaned data.
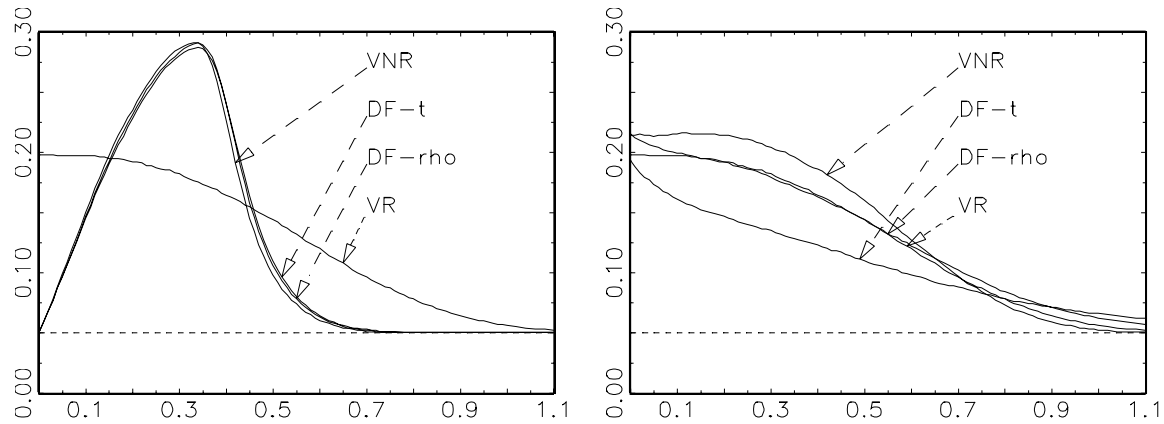

Figure 5: Size of the unit root tests in the one-limit case for various values of $-\underline{c}$. Left panel: deviations from the initial value. Right panel: demeaned data. See Figure 4 for test definitions. 

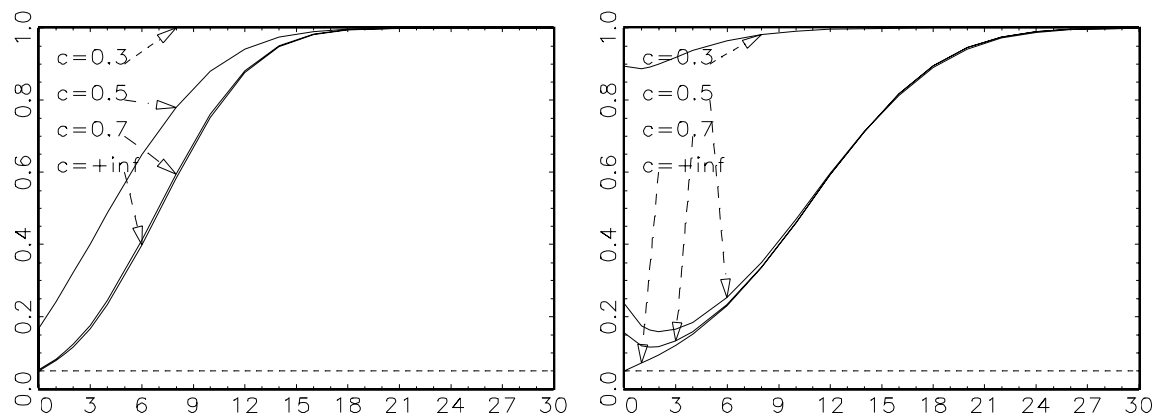

Figure 6: Asymptotic power of the unit root $Z_{\rho}$ test for various values of $\bar{c}=-\underline{c}=c$. Left panel: deviations from the initial value. Right panel: demeaned data.
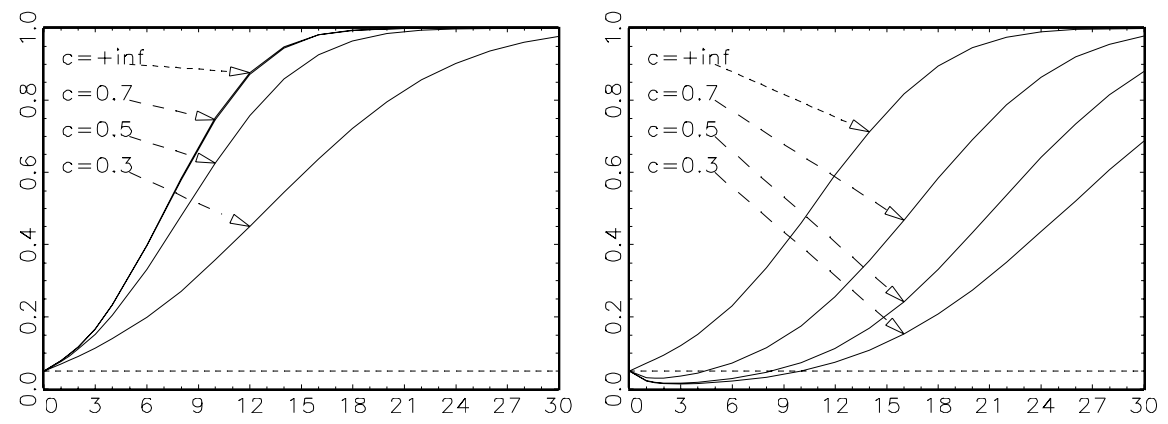

Figure 7: Asymptotic power of the 'bounded unit root' $Z_{\rho}$ test for various values of $\bar{c}=-\underline{c}=c$. Left panel: deviations from the initial value. Right panel: demeaned data. 

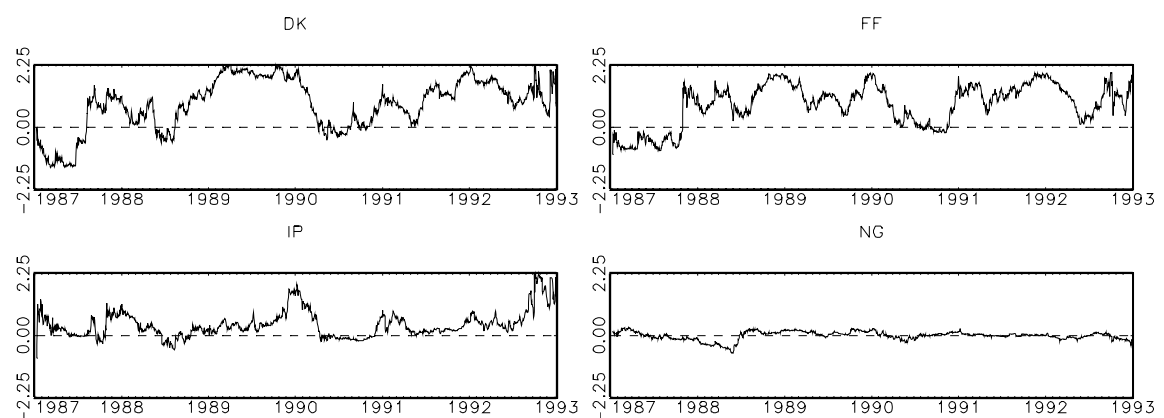

Figure 8: EMS exchange rates of the Danish Krone (DK), the French Franc (FF), the Irish Pound (IP) and the Dutch Guilder (NL) against the Deutsche Mark, 1987:01-1993:01. Percentage deviations from the central parity. 